\title{
Communications
}

This journal welcomes comments from all those involved in artificial organs as researchers, developers, or users. We would like to hear of your experiences. Please send all Communications to the Editorial Office.

\section{Abstracts from the Seventh International Workshop on Functional Electrical Stimulation September 12-15, 2001, Vienna, Austria}

EFFECTS OF TRAINING WITH ELECTRICAL STIMULATION ON KNEE JOINT TORQUE. Thomas Angeli and Thomas Denkmeier. Institute of Machine Elements and Machine Design, University of Technology, Vienna, Austria

The aim of this study was to investigate the influence of an 8 week electrical stimulation (ES) training program on the knee joint torque of the knee joint extensor muscles of 6 test persons (3 trained hobby sportsmen [T ES] and 3 untrained test persons [UT ES]). ES sessions were carried out with simultaneous maximum voluntary isometric contraction 5 times weekly. A control group of 6 test persons ( 3 T NoES and 3 UT NoES) completed the same training program without ES. Every 2 weeks measurements on a dynamometer were done before, during, and after training sessions. The averaged strength increase of the UT ES group amounted to $9.5 \%$ and of the T ES group to $5.7 \%$ at the knee joint flexion angle of $60^{\circ}$. The UT NoES group achieved $9.1 \%$ at the same knee joint flexion angle.

VARIABLE FUNCTIONAL ELECTRICAL STIMULATION DEPENDING ON KNEE FLEXION ANGLE. T. Angeli, M. Gföhler, and A. Karg. Institute for Machine Elements and Machine Design, University of Technology, Vienna, Austria

To clarify the different results of our simulation and functional electrical stimulation (FES) cycling tests, measurements on a knee dynamometer were made. The $\mathrm{m}$. quadriceps of 16 healthy test persons was activated both by FES and voluntary contraction. Stimulated with the same level of intensity in a knee flexion angle range from $5^{\circ}$ to $105^{\circ}$, the diagrams showed a very unusual course. The knee torque showed its maximum at the knee flexion angle of approximately $30^{\circ}$. Additional isometric measurements using stimulation intensity on constant on-verge-to pain levels for different knee angles were made. The measured courses of the resulting knee torque as a function of the knee angle are much closer to the results of physiologically activated muscle. These measurements show that for optimum power release, the stimulation intensity must be regulated depending on the knee flexion angle.

SECOND GENERATION MICROSTIMULATOR. I. Arcos, K. Fey, D. Mishler, D. Sanderson, C. Tanacs, M.J. Vogel, R. Wolf, Y. Zilberman, R. Davis, and J. Schulman. Alfred E. Mann Foundation, Santa Clarita, California, U.S.A.

The Alfred E. Mann Foundation has developed a ceramiccased radio frequency-driven implantable microstimulator based on the original device developed by an NIH-funded consortium. The first generation was a glass-encased injectable microstimulator with an external tantalum capacitor electrode. The secondgeneration device is a ceramic cylinder approximately 10 times stronger than the glass case with platinum electrodes and an unexposed hermetically sealed capacitor that cannot be inadvertent- ly damaged by a steel scalpel. This microstimulator, referred to as Bion, is the main component of a 255 channel wireless stimulating system, where each stimulator can be implanted via a trocar-based implant tool (developed by Advanced Bionics, Sylmar, CA, U.S.A.) or via surgical opening. The electronics module has passed a $1,000 \mathrm{~h} 125^{\circ} \mathrm{C}$ fully powered burn-in qualification test. Bion microstimulators have been implanted in rat hindlimbs for periods of up to 5 months. During the implantation time, stimulation to produce muscle twitches was applied for $53 \mathrm{~h} / 103$ days. Histological results showed benign tissue reactions resulting in identical encapsulation around Bion stimulators and passive controls. Threshold levels were measured during the stimulation period and did not change significantly over time. We have decided to develop Bion applications by seeking collaboration with the research community that could result in much wider access to this technology. We have created an online community to share and exchange information and to improve functional electrical stimulation by taking advantage of the Bion technology. We call this community Bion Technology Partnership or BionTech.

DERMATOME ELECTRICAL STIMULATION AS A THERAPEUTIC AMBULATORY AID FOR INCOMPLETE SPINAL CORD INJURED PATIENTS. Tadej Bajd ${ }^{1}$ and Helena Benko. ${ }^{2}{ }^{1}$ Faculty of Electrical Engineering, University of Ljubljana; and ${ }^{2}$ Rehabilitation Institute, Ljubljana, Slovenia

In the last decades more incomplete than complete spinal cord injured (SCI) patients are arriving in the spinal units. It is our observation that functional electrical stimulation (FES) training of their paralyzed and atrophied muscles is effective in the great majority of the patients in the early period of the rehabilitation process. The candidates are all patients with upper motor neuron lesions, in more clinical terms patients with thoracic or cervical lesions to the spinal cord. The voluntary response in partially paralyzed muscles improved in most of the patients who were treated with cyclical electrical stimulation. However, it should be stressed that FES represents 1 of the rare rehabilitative approaches for incompletely paralyzed subjects soon after accident, not only returning them to standing position but also restoring their walking patterns. Walking of incomplete SCI patients can be significantly improved by the use of simple single-channel peroneal stimulation resulting in flexion response of the paralyzed extremity. The swing phase of walking can be influenced efficiently also through cutaneous stimulation of selected dermatomes. The electrodes were placed over L-3,4 dermatome, 1 medially below the knee and the other laterally above it, with the aim to decrease the extensor spasticity of the knee extensors (innervated from the same spinal cord level as the dermatome). A stimulation frequency of $100 \mathrm{~Hz}$ and a pulse duration of $0.3 \mathrm{~ms}$ were used. The electrical stimulation did not causing any muscle contraction. Sensory electrical stimulation was delivered predominantly through the large diameter afferent fibers. Strong extensor spasticity can be observed often in the lower extremities of incomplete SCI patients. After loading the paralyzed limb during the stance phase of walking, the patients have difficulty breaking this extension tone and cannot initiate the step. The stimulation of the L-3,4 dermatome proved to be efficient in diminishing the extensor activity. Hip and knee flexion and ankle dorsiflexion wereincreased significantly when delivering the dermatome stimulation. Deramatome stimulation proved to be specially efficient when combined with treadmill walking. 
BILATERAL COCHLEAR IMPLANTATION. W.D. Baumgartner, J. Hamzavi, C. Vasak, B. Schenk, and S. Pok. Department of Otorhinolaryngology, University of Vienna, Vienna, Austria

For decades bilateral hearing aid support for children was not discussed. The importance of bilateral hearing for the development of children is quite clear, but bilateral cochlear implants are still rare because of different reasons. The aim of this study was to determine if and when a bilateral cochlear implantation is helpful and indicated. The idea of bilateral implantation is not sharp enough, and after analysis of different groups of bilaterally implanted patients, we see what we can expect for a patient's individual circumstances. From 199518 patients ( 6 adults and 12 children) underwent bilateral cochlear implantation in a 2 course surgery. Analyzing the results we decided to divide bilaterally implanted patients into 4 groups:

Group A: analog system/ multichannel continuous interleaved sampling stimulator,

Group B: different devices (for example, nucleus mini 22/med el combi 40+),

Group C: same implant type but different fitting parameter, because of etiology or surgical circumstances, and

Group D: same implant bilaterally and symmetric fitting parameter

As a result, significant superior speech understanding (especially in noise) and acoustic orientation can be achieved only by bilateral cochlear implantation in a Group D patient. To obtain a subjective (and in performance evident) benefit of a bilateral cochlear implantation, 2 multichannel fast stimulators in quite symmetric fitting parameters are mandatory. Otherwise the better ear remains dominant. For the intended benefit of bilateral implantation, we recommend bilateral cochlear implantation in a Group D situation only.

THE VIENNA FUNCTIONAL ELECTRICAL STIMULATION SYSTEM FOR RESTORATION OF WALKING FUNCTIONS IN SPASTIC PARAPLEGIA. M. Bijak ${ }^{1} \mathrm{~W}$. Mayr, ${ }^{1} \mathrm{C}$. Hofer, ${ }^{2}$ H. Lanmueller, ${ }^{1}$ M. Rakos, ${ }^{1}$ D. Rafolt, ${ }^{1}$ M. Reichel, ${ }^{1}$ S. Sauermann, ${ }^{1}$ C. Schmutterer,${ }^{1}$ E. Unger ${ }^{1}$ M. Russold, ${ }^{1}$ and $\mathrm{H}$. Kern. ${ }^{2}$ Department of Biomedical Engineering and Physics, University of Vienna; and ${ }^{2}$ Department of Physical Medicine and Rehabilitation, Wilhelminenspital, Vienna, Austria

Stimulation of leg muscles in spastic paraplegia to restore standing up from a wheelchair, stepping, and sitting down in a wheelchair is a well-known functional electrical stimulation (FES) application. Muscle activation with implanted stimulators via nerve and motor point attached electrodes or with external stimulators via surface electrodes are state of the art. Surface stimulation needs careful system setup before use, has a limit in the amount of useful channels, and a worse muscle selectivity in comparison to implanted systems but does not require any surgical intervention. We concentrated on an improved 8 channel surface stimulation system designed for easy handling and with a wide stimulation parameter range to optimize leg movement by parameter optimization. The introduced system consists of the patient wearing 4 channel stimulators (1 for each leg), a control device with batteries, power supply and bus management circuitry, and a Windows CE based palmtop computer. A special software package, installed on a standard PC or laptop computer in the rehabilitation center, allows us to devise and optimize all stimulation sequences. Finally, the results are stored in a patient related database and transferred to the palmtop computer via a database synchronization link. A wireless connection to the walker or crutch mounted control elements completes the stimulation system. First tests with patients used to FES showed smoother movements during stepping and acceptable device handling. In combination with the PC software the required stimulation sequences could be created in a very short time.
EFFECTS OF NEUROMUSCULAR ELECTRICAL STIMULATION IN PATIENTS WITH REFRACTORY HEART FAILURE. M. Bijak, ${ }^{1}$ W. Mayr,${ }^{1}$ M. Quittan, ${ }^{2}$ A. Sochor, ${ }^{2}$ R. Pacher, ${ }^{3}$ E. Unger, ${ }^{1}$ D. Rafolt, ${ }^{1}$ H. Lanmueller, ${ }^{1} \mathrm{~S}$. Sauermann, ${ }^{1}$ and G. Freilinger. ${ }^{4}$ Department of Biomedical Engineering and Physics, ${ }^{2}$ Department of Physical Medicine and Rehabilitation, ${ }^{3}$ Department of Radiology, and ${ }^{4}$ Department of Plastic and Reconstructive Surgery, University of Vienna, University Hospital (AKH), Vienna, Austria

Skeletal muscle strength and mass are severely impaired in patients with chronic heart failure. The aim of the study was to determine the impact of neuromuscular electrical stimulation (NMES) on the thigh muscles of this group of patients. Forty-two subjects with stable disease course were assigned randomly to a stimulation group (SG, mean age 59 years) and a control group (CG, mean age 57 years). A modified version of a space approved 8 channel electrical stimulation device was used to exercise SG for 8 weeks. Control parameters were isometric and isokinetic thigh muscle strength and muscle cross sectional area (CSA). An increase in muscle strength of $22.7 \%$ for knee extensors and $35.4 \%$ for knee flexors could be demonstrated in SG while CG remained unchanged or decreased by $8.4 \%$ in extensor strength. CSA increased in SG by $15.5 \%$ and in CG by $1.7 \%$. NMES of thigh muscles in patients with refractory heart failure is effective in increasing muscle strength and bulk.

THE HEMODYNAMIC FUNCTION OF INTRATHORACIC SKELETAL MUSCLE VENTRICLES AFTER RECOVERY FROM SURGERY IN PIGS. Massimo Capoccia, Hazel Sutherland, Stanley Salmons, and Jonathan C. Jarvis. Department of Human Anatomy and Cell Biology, University of Liverpool, Liverpool, U.K.

The serious shortage of donor organs for heart transplantation highlights the need for alternatives for the treatment of end-stage heart failure. A promising experimental approach is the use of skeletal muscle ventricles (SMVs) connected to the circulation to provide ventricular assistance. We present a new recovery model in pigs. The aim was to form an intrathoracic pumping chamber from the latissimus dorsi muscle (LDM) and to connect it to the descending thoracic aorta in a single surgical procedure without compromising the host circulation. The LDM was stimulated at 1 $\mathrm{Hz}$ for 4 weeks to transform the muscle to a fatigue-resistant type. The left LDM was then fashioned into a pumping chamber around a preformed lining comprising the pulmonary artery and part of the descending thoracic aorta of a donor pig. The SMV conduit was connected to the descending aorta. An R wave synchronous pulse train stimulator (LD Pace II, CCC Uruguay) was programmed to deliver a burst of impulses at $33 \mathrm{~Hz}$ every third left ventricular diastole (1:3 assist ratio). At an elective terminal procedure after 1 week transducers were placed to measure left ventricle pressure and volume, SMV pressure and volume, SMV and aortic arch pressure, and flow in the aortic root and in the SMV conduit. In 2 animals the SMV was still pumping but the aortic homograft was kinked, resulting in thrombus formation. Hemodynamic measurements were made from 1 of these SMVs after the removal of thrombus and reconfiguration of the conduit. In the third the problem was avoided by constructing the SMV closer to the aortic arch with a shorter conduit. The SMV was contractile and free from thrombus after pumping in circulation for 1 week. Activation of the SMV from the end of systole for $80 \%$ of diastole increased mean diastolic blood pressure above control levels (obtained with the SMV off) by $11.2 \pm 1.6 \%$ in 1 animal and by $15.8 \pm 0.3 \%$ in the other. Peak diastolic aortic pressure was increased by $16.9 \pm 1.4 \%$ and $20.1 \pm 1.2 \%$. The left ventricular stroke work in the post-assisted beat was decreased by $8.7 \pm 5.8 \%$ and $10.1 \pm 2.2 \%$ and the overall stroke work by $7.4 \pm$ $1.2 \%$ and $9.4 \pm 0.8 \%$. The use of the composite homograft was the key to forming and connecting the SMV in a single procedure. It has the further beneficial effect of reducing the effective preload 
for the SMV and thus decreases the risk of ischemia of the inner wall. The functional role of the homograft lining could be taken on in clinical practice by a synthetic composite or by a tissue lining produced in culture.

A TASK ORIENTED APPROACH BY MEANS OF A TRANSCUTANEOUS NEUROPROSTHESIS: A SEVEN YEAR CLINICAL EXPERIENCE FOR EXERCISE, STANDING, AND WALKING ON SPINAL CORD INJURED SUBJECTS. H.A. Cerrel-Bazo ${ }_{1}^{1}$ A. Rizzetto, ${ }^{1}$ E. Corà,${ }^{1}$ R. Bogoni,${ }^{1}$ A. Bolner, and S.W. Brown. ${ }^{2}{ }^{1}$ Centro di Neuroriabilitazione e Ricerca di Villa Margherita, Arcugnano, Vicenza, Italy; and ${ }^{2}$ Metropolitan State Hospital, Norwalk, California, U.S.A.

The aims of the study were to train spinal cord injured (SCI) individuals to perform a task by means of functional electrical stimulation FES and analyze metabolic and physiological responses, energy efficiency, and performance of the SCI standing and walking by means of a transcutaneous FES system (TFES). Sixty motor complete SCI were able to stand and walk following a computer-controlled FES exercise program (CCE) and a gait training program (GTP) by TFES. Under standard conditions the above mention variables were recorded while standing (tilt table and by TFES) for $30 \mathrm{~min}$ and walking (on a treadmill) for $6 \mathrm{~min}$ (at 5, 10, 15, 18, and $20 \mathrm{~m} / \mathrm{min}$ speed) and for $45 \mathrm{~min}$ (at self preferred maximum speed [SPMS]). All data were entered into a Statview SE+ program for computers. ANOVA was used to assess energy cost between groups (the TFES group was compared to a control group, able bodied and advanced reciprocating gait orthosis [ARGO] SCI users). CCE-GTP and the ability to stand and walk independently with TFES average 5 to 6 months. Based on standing and walking endurance TFES walkers were classified as good walkers with a speed of $14 \mathrm{~m} / \mathrm{min}$, a walking distance of 580 $\mathrm{m}$, a walking time of $42 \mathrm{~min}$, and a standing time of $30 \mathrm{~min}$. Moderate walkers had a speed of $10 \mathrm{~m} / \mathrm{min}$, a walking distance of $160 \mathrm{~m}$, a walking time of $12.5 \mathrm{~min}$, and a standing time of $23 \mathrm{~min}$. Poor walkers had a speed of $8 \mathrm{~m} / \mathrm{min}$, a walking distance of $42 \mathrm{~m}$, a walking time of $3 \mathrm{~min}$, and a standing time of $3 \mathrm{~min}$. The TFES group showed higher statiscally significant $(\mathrm{p}<0.001)$ values for most variables analyzed when compared to the control group. Oxygen cost increased one-fold from sitting to TFES standing and three- to four-fold when TFES walking. At SPMS 45 min walking posthoc analysis showed a higher metabolic efficiency for the able bodied group compared to the TFES and ARGO groups. During standing and walking metabolic and physiological responses were significantly higher for the TFES group. Statiscally significant differences were reported for lactic acid, $\mathrm{O}_{2}$ pulse, ventilation, and catecholamines, higher for the TFES group.The TFES mean total sweat rate was higher and statistically significant compared to the able bodied group. Well selected and trained SCI patients can perform a task oriented approach by means of TFES. Functional achievment was not the goal. Treadmill walking at a costant speed is metabolically demanding. Good TFES walkers adapt their cardiovascular and pulmonary responses to the physical effort. Good standing and walking performances appear to be correlated to a higher metabolic efficiency. Daily changes of the SCI system need to be studied to better address the problem of efficient continuative performance. TFES standing and walking are good active physical therapies for well-selected SCI subjects.

QUALITY PRODUCTS AND QUALITY FOR PATIENTS: ISO9002 FOR MANUFACTURE AND CLINICAL SERVICE. S.E. Crook, I.D. Swain, P.N. Taylor, and S. Finn. Department of Medical Physics and Biomedical Engineering, Salisbury District Hospital, Salisbury, U.K.

As professionals working in the field of functional electrical stimulation (FES) it is necessary to achieve and maintain high standards. There may also be requirements from legislation or recommendations from professional bodies. In the case of manufacturing of medical devices there is a European medical devices directive that has been made law by European Community member states. This requires stimulation devices to carry a CE mark and the manufacture must therefore take place under a quality system that has been assessed by a notified body. The decision was taken in Salisbury that we would cover the patient treatment process as well as medical device manufacture within the scope of our departmental quality management system. A quality manager was appointed and some help from a consultant was enlisted in order to compile the procedures necessary. However, approval for the system only would be achieved if it could be demonstrated that the system was being followed. The procedures are designed to generate records that can be checked by internal management and an external inspector. The equipment manufacture was given highest priority to enable continued sales outside of our hospital and approval for ISO9002/46002 was awarded in October 1998. ISO9000 was written for use in the manufacturing industry and not the clinic. However, it was possible to generate a set of procedures that would track the appointment and treatment process. In order to demonstrate the effectiveness of our treatment using the Odstock dropped foot stimulator data was collected on forms that were filled in at each patient attendance. Completion of the forms was brought into the quality system and data could be used for scientific papers as well as treatment or equipment improvements. At all stages a failure in the process or a complaint would generate a form to be considered and acted upon at regular management meetings. Internal audit of the quality system ensured that the procedures were being followed and inappropriate procedures were modified. In due course the clinical service was brought into the scope of our ISO9002 approval in early 1999.

PARAPLEGIA: THE IMPLANTABLE PRAXIS FES-24B SYSTEM FOR FUNCTIONAL RESTORATION. Ross Davis, ${ }^{1}$ Andrew Barriskill, ${ }^{2}$ Zoran Milijasevic, ${ }^{2}$ Thierry Houdayer, ${ }^{1}$ and Brian Andrews. ${ }^{3}{ }^{1}$ Neural Engineering Clinic, Augusta, Maine, U.S.A.; ${ }^{2}$ Neopraxis Pty. Ltd., Lane Cove, N.S.W., Australia; and ${ }^{3}$ National Spinal Injury Center, Stoke Mandeville Hospital, Aylesbury, U.K.

Neopraxis Pty. Ltd. (Lane Cove, N.S.W., Australia) is manufacturing the new implantable Praxis FES24-B System to provide multiple benefits to suitable paraplegic users such as bladder control, upright functional mobility, pressure relief, and lower extremity exercise. The implant achieves these functions through epineural stimulation of nerves in the legs, the medial lumbar region, the sacrum, and the conus medullaris. A body worn controller, the Navigator, transcutaneously powers and controls an implanted stimulator via a magnetically held transmit coil/ antenna. The stimulator is connected to 22 electrodes by flexible and stretchable insulated leads. Sensor packs attached to each thigh and shank and the trunk provide the Navigator's software strategies with real time information on the position of the lower extremities and the trunk. To assist simple locomotor functions we will focus on how the system can complement the use of a wheelchair and be helpful in overcoming obstacles to wheelchair access especially doorsteps and unadapted bathroom facilities. In addition, being able to stand up to reach objects and perform prolonged manual tasks would be convenient for many workplace and home situations. The FES24-B stimulator provides up to 8 $\mathrm{mA}$ of charge-balanced stimulation current via a biphasic waveform. Pulse widths can be varied from $25 \mu$ s up to $500 \mu \mathrm{s}$. The total pulse rate can range up to 14,400 pulses per second. The stimulator provides real time data telemetry functions including the ability to measure the impedance of the current path through each electrode and the ability to transmit voltage measurements from each electrode. Since 19912 paraplegic subjects have been implanted with earlier models. A multicenter clinical trial of the system will commence in mid 2001. 
TWENTY-SEVEN YEARS OF CLINICAL EXPERIENCE WITH IMPLANTABLE NEUROPROSTHESES FOR VARIOUS APPLICATIONS. Ross Davis. Neural Engineering Clinic, Augusta, Maine, U.S.A.

Since 1973 the author has been implanting neural stimulators and drug pumps to restore or improve motor function in spinal cord and brain injury, stroke, and multiple sclerosis. Also these neural implants have been used to modulate and decrease pain (chronic pain syndromes) and spasticity (cerebral palsy, stroke, and multiple sclerosis) and to reduce chronic intractable seizures. During these 27 years, many worthwhile developments in the implantable neural equipment have been realized as well as many lessons learnt to improve operative techniques to ensure safer and improved results for the patients. With the advent of regulatory boards and increasing restrictions by health insurance companies, more challenges have arisen to allow new equipment and applications to be started, tested, and accepted for clinical use and hopefully for payment. The relationships between manufacturers and concept originators have varied over the years. Often, personalities can make or break the developmental process. The increasing needs to patent ideas and intellectual properties, which sometimes have been around for years and even previously published (and missed by the patent officer), have led to vigorous legal battles, consumed money with time delays, or the result of surrendering worthwhile projects. There is a need for a responsible independent appeals board made up of senior experienced researchers to review these disputed intellectual property claims. Then their findings should be admissible in court if the case should reach this stage. Much has been accomplished in the neuroprosthesis area by the expanding presence of biomedical engineers, more scientifically trained physicians and therapists, and successful biomedical manufacturers. Because of the previous and continuing investments by private Individuals, universities, research foundations, and private and government granting bodies, the future for neuroprosthetic device development and use continues to look very bright.

THE MiVIP VISUAL PROSTHESIS FOR OPTIC NERVE STIMULATION. J. Delbeke, ${ }^{1}$ M.-C. Wanet-Defalque, ${ }^{1}$ B. Gérard, ${ }^{1} \mathrm{M}$. Troosters, ${ }^{2}$ G. Michaux, ${ }^{1}$ and C. Veraart. ${ }^{1}{ }^{1}$ Universite de Louvain, Neural Rehabilitation Engineering Lab, Brussels; and ${ }^{2}$ NeuroTech S.A., Louvain-la-Neuve, Belgium

The purpose of the present experiment was to evaluate psychophysically the implantee's benefits when using the MiViP optic nerve visual prosthesis. A 59 year old volunteer with retinitis pigmentosa was implanted initially with a self-sizing spiral cuff electrode around her right optic nerve in February 1998 and then with an implanted stimulator and antenna for telemetry in August 2000. The silicone rubber spiral cuff electrode includes 4 platinum contacts which are driven by independent current sources in the implanted stimulator. Biphasic pulses with charge recuperation have a time resolution of $21.3 \mu$ s and a current intensity ranging from $10 \mu \mathrm{A}$ to $3 \mathrm{~mA}$ with a nonlinear amplitude resolution. Optic nerve activation is achieved under control of external equipment using radiofrequency transmission with a $3 \mathrm{Mbit} / \mathrm{s}$ data rate. External equipment includes a dedicated head-worn artificial retina and processors. Image processing extracts pixels from camera images and defines appropriate stimulating conditions of the nerve susceptible to elicit corresponding phosphenes in real time. Psychophysical experiments included a pattern recognition task. Fifty simple patterns were used during a 10 session program with feedback from the instructor. Four evaluation sessions were embedded in the training program to assess learning improvement. This project fully complies with the Declaration of Helsinki, and was approved by the ethics committee of the School of Medicine and University Hospital of the University of Louvain, Brussels. In the pattern recognition task, the performance improved regularly with practice with an increasing score and a decreasing delay to recognition. Evaluation of general mobility improvement with the portable system is in progress. In conclusion, the results obtained demonstrate the potentials of the optic nerve visual prosthesis. A low-resolution artificial vision can be expected from the prosthesis after extensive training. The final objective of the project is to allow the volunteer to better cope with her visual environment during mobility and grasping.

ROLE OF NEUROMUSCULAR STIMULATION AND FUNCTIONAL ELECTRICAL STIMULATION IN THE PRACTICE OF NEUROREHABILITATION. Milan R. Dimitrijevic, ${ }^{1}$ and Meta M. Dimitrijevic. ${ }^{2}{ }^{1}$ Baylor College of Medicine, Houston, Texas, U.S.A.; and ${ }^{2}$ University Institute for Clinical Neurophysiology, Ljubljana, Slovenia

The physicians and their multidisciplinary teams involved in the clinical practice of neurological rehabilitation have more and more opportunities to apply neuromuscular stimulation (NMS) and functional electrical stimulation (FES) of peripheral nerves as a part of their daily practice. We outlined clinical protocols of NMS and FES in following clinical conditions of upper motor neuron dysfunction:

(1) To prevent consequences of disuse of the neuromuscular system of the upper motor neuron;

(2) To facilitate recovery processes of impaired upper motor neuron functions due acute and/or subacute neurological conditions;

(3) To maintain or enhance trophic state of the muscle;

(4) To modify altered control of muscle tone;

(5) To modify altered patterns of automatic and volitional functional movements;

(6) To enhance functional movement of the single joint muscle group within intact functional multijoint movement; and

(7) To modify altered neurocontrol of posture, locomotion, and skillful movements.

We emphasized the importance of understanding of the motor control alteration of dysfunction while developing clinical protocols and defining the goals. It is very important to be aware that similar clinical findings and due to the same cause can have different features of residual motor control and therefore potentials for recovery or modification can be very different. We conclude by providing evidence for 2 clinical values of NMS and FES practice in neurological rehabilitation programs:

(1) Evaluation of the upper motor neuron dysfunction and altered motor control of impaired movements, and

(2) Externally controlled facilitation of residual neurocontrol of movements as well as maintenance of optimal functional status of residual neurocontrol when recovery processes are not present.

USING FUNCTIONAL ELECTRICAL STIMULATION IN PARKINSON'S DISEASE. S.M. Finn, G.E. Mann, and P.N. Taylor. Department of Medical Physics and Biomedical Engineering, Salisbury District Hospital, Salisbury, U.K.

Parkinson's disease (PD) affects $1 \%$ of people over 50 years of age and is a leading cause of disability in those over 60 years of age. Characteristic neurological impairments in PD include resting tremor, rigidity, slowness of movement (bradykinesia), and postural instability. Walking becomes slower, with reduced stride length and cadence giving rise to a shuffling gait. As the disease progresses there are difficulties in initiating and maintaining movement, resulting in freezing (akinesia), which occurs particularly in confined spaces. There is evidence that external cues (visual, auditory, cognitive or sensory) may be able to compensate for the defective internal cueing system improving step initiation, force production, and walking speed in PD patients. At present, 3 case studies have been conducted to investigate the effects of 
functional electrical stimulation (FES) on these parameters of gait. All have a long history of PD. Initially the single channel Odstock dropped foot stimulator (ODFS) was set up to stimulate the common peroneal nerve on the most affected side, in order to become used to the effects of stimulation and to use of the stimulator. After 3 months the subjects progressed to bilateral dropped foot stimulation from a 2 channel ODFS in order to provide a bilateral sensory cue. The subjects were observed walking with and without stimulation and with and without medication. Single channel common peroneal stimulation was effective in producing improved heel strike and reducing shuffle as the patient approached and passed through doorways. The subjects felt that the stimulation gave greater confidence in walking and that bilateral stimulation gave greater symmetry. The subjects appeared to benefit from the use of FES. This may be due to the sensation of stimulation acting as a triggering external sensory cue, to increased proprioceptive input from the contraction of the anterior tibial muscles, or a combination of the two.

A PATIENT'S VIEW OF FUNCTIONAL ELECTRICAL STIMULATION CYCLING EXERCISE. R. Fitzwater. Royal National Orthopaedic Hospital, Stanmore, U.K.

This presentation was made by a general medical practitioner who has had an imcomplete T11/12 paraplegia since 1988 and from December 1998 has been involved in a surface functional electrical stimulation (FES) cycling research program. Since the first report (1983) on the use of FES to enable a spinal cord injured (SCI) patient to achieve leg powered cycling on a tricycle, FES cycling exercise has mainly been carried out statically on cycle ergometers in clinics or laboratories. Even the moderate levels of exercise possible with such an approach are reported to have given many health benefits. After nearly 20 years of this field, it is high time the technique moved out of the laboratory and into SCI patients' everyday lives, where it would be more convenient to achieve the higher levels of exercise that could give the greatest benefit. The personal value and benefits of of being involved in over 2 years of FES cycling exercise include the expected increases in leg muscle strength, endurance, and thickness. Psychological mood has improved. Spasms are less troublesome. Voluntary function has increased. The main disadvantage of being involved in this FES research program is the time taken. Something must be done to speed up the process of donning and doffing all the electrodes and wires. Also transfer to and from the tricycle must be made easier. There is insufficient power available from the FES to keep up with able bodied cyclists, yet more than enough to leave an able bodied walking companion far behind. The effort involved for most SCI patients to walk even 1 kilometer is huge. Wheels are efficient. Real outdoor functional leg locomotion is feasible for this subject using a recumbent tricycle as a wheeled orthosis.

USABILITY OF ELECTROENCEPHALOGRAM SIGNALS FOR FUNCTIONAL ELECTRICAL STIMULATION CONTROL: THROUGH EVENT RELATED POTENTIALS CAUSED BY OBSCURE PICTURES. Mariko F. Funada,${ }^{1} \mathrm{Sa}-$ toshi Suzuki, ${ }^{2}$ Keiko Kasamastu, ${ }^{2}$ Miki Shibukawa, ${ }^{2}$ and Satoki P. Ninomija. ${ }^{2}{ }^{1}$ Hakuoh University, Tochigi;and ${ }^{2}$ Aoyama Gakuin University, Chitosedai, Tokyo, Japan

The goal of this article is to characterize human recognition mechanisms when people watch some obscure pictures through electroencephalograms (EEGs), especially event related potentials (ERPs). ERPs are special potentials measured from EEGs and caused by special events like watching some pictures, listening to some sounds, or feeling some pain, and so forth. Since the brains of human beings control their recognitions, judgments, and activities and EEGs or ERPs explain directly the states of brains, EEGs or ERPs are one of the most excellent indicators that show the dynamic states of brains. Our approaches were to design an experiment that was to judge obscure pictures on a computer screen as correct or not, to execute the experiment, and to record EEGs during the experiment. Then we analyzed the recorded EEGs and obtained single stimulated ERPs. The changes of ERPs consist of at least 2 different components; one is background activity of EEGs and the other is the change of ERPs. We tried to separate these components from each other and made a model to explain the results of the separations. Using the model, we characterized the human recognition mechanism when people watch some obscure pictures.

FUNCTIONAL ELECTRICAL STIMULATION CYCLING: MEASUREMENTS AND INDIVIDUAL ADAPTATION OF STIMULATION PATTERNS ON A TEST BED AND A MOBILE TRICYCLE. M. Gföhler ${ }^{1}{ }^{T}$. Angeli, ${ }^{1}$ P. Lugner, ${ }^{2}$ C. Hofer, ${ }^{3}$ M. Bijak, ${ }^{4}$ M. Reichel, ${ }^{4}$ and W. Mayr ${ }^{4}{ }^{1}$ Institute of Machine Elements and ${ }^{2}$ Department of Mechanics, Vienna University of Technology; ${ }^{3}$ Department of Physical Medicine, Wilhelminenspital; and ${ }^{4}$ Department of Biomedical Engineering and Physics, Vienna University Medical School, Vienna, Austria

An important motivation in studying functional electrical stimulation (FES) cycling is to combine the physiological benefits of FES with the psychological incentive of independent locomotion. As paralyzed muscles are mostly atrophied and only a limited number of effective leg muscles can be reached by surface electrodes it is important to convert the generated muscle forces into drive power with the highest possible efficiency. In this study static and dynamic measurements with paraplegic test persons on a freely adjustable test bed were performed to determine the individual FES cycling performance and to individually optimize the stimulation patterns. Hydrogel surface electrodes were used to activate quadriceps, hamstrings, and gluteus maximus, and to elicit the peronaeus reflex with a stimulation frequency of $30 \mathrm{~Hz}$ For the static measurements 20 equiangular points were defined along the pedal path. In every point the muscle was stimulated for $0.75 \mathrm{~s}$, and the pedal forces were measured by a force-measuring crank. Orientation and magnitude of the active forces (directly caused by active muscle forces) applied to the crank in the parasagittal pedaling plane were calculated for a number of geometrical positions of the rider and variations of the stimulation parameters. Out of these data it could be seen in which part of the pedal path positive drive torque was applied. Then in the dynamic measurements the muscle was stimulated in this interval, and the start and end points of the stimulations were varied to find out in which interval maximum positive drive torque could be applied. Tests with all muscles stimulated together were performed to show how much the muscles influenced each other. Finally a set of optimized parameters for stimulating all muscles together while pedaling was derived for each individual test person and tested on a specially developed mobile tricycle for paraplegics.

AN EXPERIMENTAL EVALUATION OF THE GYROSCOPE AS A SENSOR IN FUNCTIONAL ELECTRICAL STIMULATION FOOT-DROP CORRECTION SYSTEMS. S.N. Ghoussayni, ${ }^{1}$ J.R. Henty, ${ }^{1}$ D.E. Wood ${ }^{2}$ and D.J. Ewins. ${ }^{1}$ ${ }^{1}$ Biomedical Engineering Group, University of Surrey, Guildford; and ${ }^{2}$ Department of Medical Physics and Biomedical Engineering, Salisbury District Hospital, Salisbury, U.K.

This article presents the work done on the use of a gyroscope (Gyro) as a sensor for foot-drop correction systems using functional electrical stimulation (FES). These systems usually employ foot switches to control the timing of stimulation. It is believed that the replacement of the heel switch with the gyroscopic sensor would offer several advantages. The performance of the new sensor and associated software in gait event detection have been tested and compared to foot switch and kinematic data. The tests were carried out with both able-bodied and hemiplegic patients walking over different terrains. The results indicate that the Gyro 
can be used to detect the necessary gait events for controlling the stimulator timing. The replacement of the foot switch by the Gyro offers several advantages that could improve system reliability and function. The Gyro is a small and lightweight sensor (with potential of further miniaturization and implantation), which easily can be donned and doffed (positioning is not very critical) with minimal encumbrance to the patient. The nature of the Gyro contributes to its high reliability and long lifetime during which there is little or no deterioration in its performance. As a replacement for foot switches in FES foot-drop correction systems, the sensor output has to be adapted to be suitable for timing the stimulation. In our work this has been done by developing detection software for the Gyro signal to give 4 gait events (heel contact, foot flat, heel rise, and toe off). A portable microcontroller based unit with data logging capability was developed. This unit runs the gait event detection software and provides real time detection. This system was used for the evaluation of the sensor and associated software by comparing the detection times to those obtained from simultaneously recorded foot switch and kinematic data. The evaluation of the system involved its use by both ablebodied subjects and patients with foot-drop, and the system performance was tested over different terrains including level and inclined walking and staircase climbing.

THE VIENNA SKELETAL MUSCLE VENTRICLE PROJECT: FIRST RESULTS FROM CHRONIC EXPERIMENTS IN GOATS. W. Girsch, H. Lanmüller, M. Rab, L.P. Kamolz, U. Windberger, H. Schima, R. Avanessian, M. Greher, M. Ulrich, H. Beck, W. Haslik, and R. Seitelberger. Medical School, University of Vienna, Vienna, Austria

A new type of skeletal muscle ventricle (SMV) constructed of biological materials only was designed as an aortic counterpulsation device. The SMV consists of an aortic homograft enlarged to a pouch with pericardium and is anastomosed in parallel to the descending aorta. The latissimus dorsi muscle (LDM) is wrapped around the pouch. Stimulation electrodes are applicated to the thoracodorsal nerve and $\mathrm{R}$ wave triggered burst stimulation is applied during cardiac diastole. In a series of acute experiments in sheep the hemodynamic efficacy was demonstrated and a standardized surgical procedure was established. A series of chronic experiments in goats was scheduled in order to evaluate the reliability and patency of this new type of SMV and its overall influence to the circulation. In 1 goat an unconditioned LDM was used for SMV construction. In 2 goats the LDM was preconditioned by means of a newly developed fully implantable electrocardiogramtriggered multichannel stimulator and the SMV was constructed. The goats survived the procedure without any complications. The SMV was in circulation for 4,5 , and 6 months in these first animals, stimulated chronically at a rate of $1: 5$ compared with the native heart rate. Reconstitution of the resected third rib caused compression of muscle and nerve in the first animal. Consecutive loss of muscle force terminated this experiment. The second and third goat were killed as scheduled. In all 3 animals the SMV was found patent at the end of the experiment. Goats turned out to be appropriate for this kind of experiments. The configuration produced distinct hemodynamic changes required for aortic counterpulsation. Several problems seen in the first and second experiment necessitated modifications of the surgical procedure. The observed results still are promising and the experiments will be continued.

BIOMECHANICAL HEARTS MADE IN A ONE STEP OPERATION. N.W. Guldner ${ }^{1}{ }^{\text {P. Klapproth }},{ }^{1}$ M. Großherr, ${ }^{2}$ A. Brügge, ${ }^{1}$ A. Sheikhzadeh, ${ }^{3}$ R. Tölg, ${ }^{3}$ E. Rumpel, ${ }^{4}$ R. Noel, ${ }^{5}$ and H.H. Sievers. ${ }^{1}{ }^{1}$ Klinik für Herzchirurgie, ${ }^{2}$ Klinik für Anästhesiologie, ${ }^{3}$ Klinik für Kardiologie, ${ }^{4}$ Institut für Anatomie, and ${ }^{5}$ Allgemeine Tierhaltung, Medizinische Universität Lübeck, Lübeck, Germany
Up to now skeletal muscle ventricles have required a 2 step procedure; i.e., they have been integrated into blood circulation by a second operation after several weeks of vascular delay and electrical conditioning. This study investigates whether muscular blood pumps could be made in a clinically more favorable 1 step operation and could create chronically a hemodynamic relevant pumping capacity under support of Clenbuterol. In adult Boor goats $(\mathrm{n}=5)$ the latissimus dorsi muscle $(\mathrm{LDM})$ was wrapped around a polyurethane chamber that was connected to the descending aorta by 2 vascular prostheses. This flow-through pumping chamber containing a stabilizing inner layer (called biomechanical heart $[\mathrm{BMH}])$ is formed and immediately made to work against a systemic load under the support of Clenbuterol (5 \&mult; $150 \mathrm{mg} /$ week). Intraoperatively, mean stroke volume of BMHs was $53.8 \pm 22.4 \mathrm{ml}$. At follow-up, supported heart cycles were compared to nonsupported ones. After 1 month of training the left ventricle's mean diastolic (PMD) and end diastolic pressure (PED) of BMH supported cycles differed significantly from nonsupported ones (PMD $=+2.9 \pm 1.1 \%$ [ $<0.003]$, PED $=$ $-2.4 \pm 1.3 \%[\mathrm{p}<0.02])$. After supported heart contractions subsequent left heart maximal rate of pressure generation $d P / d t$ max increased by $20.5 \pm 8.1 \%(\mathrm{p}<0.0001)$. One $\mathrm{BMH}$, catheterized after 132 days of training, shifted $34.8 \mathrm{ml}$ per beat and $1.4 \mathrm{~L} / \mathrm{min}$ using an LDM of $330 \mathrm{~g}$. Depending on duration of training, percentage of myosin heavy chains Type 1 ranged between $31 \%$ and $100 \%$. Biomechanical hearts can be made in a 1 step operation. Under support of Clenbuterol they were hemodynamically effective with a remarkable volume shift and unloading of the left ventricle.

A STIMULATOR FOR FUNCTIONAL ACTIVATION OF DENERVATED MUSCLES. C. Hofer,${ }^{1}$ W. Mayr ${ }^{2}{ }^{2}$ H. Stöhr, ${ }^{2}$ and H. Kern. ${ }^{1}{ }^{1}$ Department of Physical Medicine, Wilhelminenspital; and ${ }^{2}$ Department of Biomedical Engineering and Physics, University of Vienna, Vienna, Austria

In recent years various studies proved that electrical stimulation can improve contractile capability and restore muscle function in long-term denervated degenerated muscles. The low excitability of the muscle cells at the initial stage of training and surrounding connective tissue, acting as an electrical shunt, require special stimulation parameters. Until now no appropriate devices (stimulators) were commercially available; therefore, we were forced to design our own stimulators. The control unit of the stimulators is based on a microprocessor for maximum flexibility regarding the generation of the parameters such as pulse amplitudes, pulse width, frequency, stimulation times, ramps, etc. In addition the microprocessor design allows recording of training data such as stimulation date, time, duration, and used programs. The voltage constant output stage of the stimulator is able to generate a biphasic charge balanced stimulation pulse with pulse width of 1 to $300 \mathrm{~ms}$, stimulation voltages up to $\pm 80 \mathrm{~V}\left(160 \mathrm{~V}_{\mathrm{ss}}\right)$, and $250 \mathrm{~mA}$ maximum stimulation current. To prevent direct current due to inexact charge compensation, the stimulation pulses are coupled capacitively. Simultaneous 2 channel stimulation with independent intensity is possible. The stimulators are programmed with notebook or personal digital assistants via infrared serial interface. This concept avoids stimulation with the wrong parameters because the patient only can use the stimulation parameters preprogrammed for him in the outpatient clinic. For training at home only changes in stimulation intensity within given limits are possible. The portable units are powered by an internal rechargeable battery ensuring mains isolation. Highly efficient switched voltage regulators are used for the power supply of all circuits to increase operating time of the stimulator.

PRELIMINARY ECONOMIC EVALUATION OF ELECTRICAL STIMULATION TREATMENT OF THE UPPER EXTREMITY IN POSTSTROKE HEMIPLEGIA. M.J. Ijzerman, ${ }^{1,2}$ J.R. de Kroon, ${ }^{1}$ and G.J. Renzenbrink. ${ }^{1}{ }^{1}$ Roessingh Re$\overline{\text { search }}$ and Development, Enschede; and ${ }^{2}$ Academuc Hospital 
Vrije Universiteit, Department of Rehabilitation Medicine, Amsterdam, The Netherlands

Cost-effectiveness analysis (CEA) receives increased attention as reimbursement and social justification of health care expenses is considered. Worldwide, the experience with technology assessment and CEAs on neuroprosthetics is limited. Regarding neuroprosthetics probably the most experience is available on the VOCARE bladder stimulator in spinal cord injury. Since the early seventies many studies have been published on the use of electrical stimulation to enhance motor control in poststroke hemiplegia. A few years ago some commercial systems became available. Cost effectiveness of the treatment is expected to be useful while negotiating with insurance companies. It was argued that an economic evaluation should be designed according to the stage of clinical investigation. Most of the trials on electrical stimulation devices can be considered as Stage 2 trials. Therefore, the current study was carried out to gain insight into the economic potential of electrical stimulation treatment in poststroke hemiplegia. The study was carried out using a hypothetical treatment ( $>3$ months for $3 \mathrm{~h}$ a day) of a chronic stroke patient with a spastic hemiparesis. Relevant costs associated with stroke (direct and indirect medical costs and productivity loss) were determined using a database of the Dutch Health Insurance Council (1997). Costs of the treatment (therapist and equipment) were incorporated. The effects of electrical stimulation (ES) on resource use (e.g., reduction in medical consumption) were estimated using a standardized questionnaire. The questionnaire was sent to investigators (7 physicians for physical medicine and rehabilitation and 7 therapists) who previously participated in a multicenter trial on ES in poststroke hemiplegia. A contingent valuation (willingness to pay [WTP]) approach was used to obtain estimates of the monetary value of the treatment outcome (O'Brien et al., 1996). WTP was obtained for 3 different probabilities $(10,50$, and 100\%) of a successful outcome. Success was defined as reduced arm spasticity, slightly less edema, less shoulder pain, and a moderately improved hand function. WTP estimates were obtained from the experienced clinicians and a group of nonexperienced reference population $(\mathrm{n}=20)$. Experienced clinicians expected that ES treatment would not reduce health care expenses, except for the use of spasmolytics and physical therapy. Use of ES devices would increase the costs of treatment of a stroke patient with approximately DFL. 5000.- assuming at least 2 years of use. A systematic review of randomized controlled trials showed a moderate evidence of effectiveness of ES on motor control. The reviewed (intermediate) outcome measures typically cannot be used for an economic evaluation. WTP of a reference population was approximately DFL. 5230.- assuming a 100\% probability of success. WTPs assuming a $50 \%$ and $10 \%$ probability were DFL. 3970.- and DFL. 2100.- respectively. There is no doubt that a CEA alongside a clinical trial is required in order to show cost effectiveness of ES. The present study, however, suggests that such a CEA is useful; i.e., ES treatment may be cost effective if the probability of success is nearly $100 \%$. It means that a critical examination of the patient is required before an ES device is prescribed to a patient. Several methodological problems need discussion in the current study. Outcome valuation using a WTP approach is relatively new and not without discussion. We expect however that the WTP approach will be more useful compared to other outcome measures because this approach might be more responsive to change.

SLIDING MODE CONTROL OF FUNCTIONAL ELECTRICAL STIMULATION FOR KNEE JOINT ANGLE TRACKING. Sašo Jezernik,${ }^{1}$ Philipp Inderbitzin,${ }^{1}$ Thierry Keller, ${ }^{1}$ and Robert Riener. ${ }^{2}{ }^{1}$ ETH Zürich, Automatic Control Laboratory, Zürich, Switzerland; and ${ }^{2}$ Technical University of Munich, Institute of Automatic Control Engineering, Munich, Germany

Functional electrical stimulation (FES) can be used to restore movement in certain paralyzed individuals by stimulation of intact peripheral nerves. Stimulation produces muscle contractions and generates joint movements. The underlying physiological/ biomechanical system is highly nonlinear and time variant, and a feedback control strategy is necessary for satisfactory control of the joint angles. However, many classical closed-loop control algorithms were found unable to provide an adequate movement control. The purpose of our research is to develop new FES control strategies that will insure stable angle tracking with an improved performance and that could be applied for general movement control like grasping, reaching, and walking. In the present study, an inherently robust control strategy called sliding mode was applied to the problem of knee joint angle tracking by stimulation of the quadriceps muscles. The controller was synthesized using a state-space model of a human knee and muscle (composed of activation dynamics, muscle dynamics, and biomechanics). The model was experimentally validated in our earlier studies. The derived sliding mode control law provided asymptotic stability of the knee joint angle and velocity and was robust to the muscle fatigue. It was derived in a closed form by considering only a subsystem of the muscle-knee model and using a joint angle-joint velocity sliding surface stabilized by appropriately generated muscle activation that followed from the sliding mode reachability and stabilizability conditions. In our earlier studies, instead of using a closed-form expression for the sliding mode controller, we only used an approximate sliding mode control law, and additionally necessitated a cascaded proportional-integral (PI) controller, which degraded the performance. Simulations with the new controller were carried out in SIMULINK with different knee angle trajectories and different controller parameters, and the results were evaluated. These simulation studies will be followed by human experiments.

A PILOT STUDY TO INVESTIGATE THE COMBINED EFFECT OF BOTULINUM NEUROTOXIN TYPE A AND FUNCTIONAL ELECTRICAL STIMULATION WITH PHYSIOTHERAPY IN THE TREATMENT OF SPASTIC DROPPED FOOT IN SUBACUTE STROKE. C.A. Johnson, ${ }^{1}$ I.D. Swain, ${ }^{1}$ D.E. Wood, ${ }^{1}$ D. Bürhs, ${ }^{1}$ and J.H. Burridge. ${ }^{2}{ }^{1}$ Department of Medical Physics and Biomedical Engineering, Salisbury District Hospital, Salisbury; and ${ }^{2}$ University of Southampton, Southampton, U.K.

The design was a preliminary randomized controlled trial. The subjects were observed over a period of 16 weeks, including an initial 4 week baseline phase to establish each patient's rate of change. Assessments were carried out at Weeks -4, -2, 0, 2, 4, 8, and 12 . All patients received physiotherapy; the treatment group (TG) also received botulinum neurotoxin Type A (BoNTA) at Week 0 and functional electrical stimulation (FES) during the subsequent 12 weeks of the study period. Nineteen patients were studied who had a spastic dropped foot following a stroke, identified by clinical examination and surface, kinetic, electromyography (EMG). Premature calf activation during the stance phase of walking was present in all subjects. All patients were within 1 year of their stroke and ambulant over $10 \mathrm{~m}$, with a stick and/or stand by supervision if required. Patients received physiotherapy locally, at least 3 times per week as an in-patient and twice a week as an out-patient. At the end of the baseline period the subjects in TG were given BoNTA to the medial and lateral heads of gastrocnemius and tibialis posterior, and set up with an Odstock Dropped Foot Stimulator III (ODFSIII). Subjects in TG used the ODFS III on a daily basis to assist their walking. The physiotherapists who were treating the patients on the trial recorded their physiotherapy treatment activity and any significant events in the patients' rehabilitation. All subject assessments for the trial were carried out independently by the research physiotherapist. Primary outcome measures were walking speed $(\mathrm{m} / \mathrm{s})$ and Physiological Cost Index (PCI) of gait. Other measures included the Rivermead Motor Assessment Scale, Modified Ashworth Scale, testing reflexes and scoring clonus, Barthel Index, SF36 Health 
Survey, and a semistructured interview. Surface, kinetic EMG was used to record activity of the tibialis anterior and the lateral head of the gastrocnemius. Preliminary results have been calculated for the primary outcome measures of walking speed and PCI. Regression lines for nonstimulated walking speed in both groups follow a broadly upward trend that is highly significant $(\mathrm{CG}, \mathrm{p}=$ 0.002 ; TG, $p=0.001)$. There was a highly significant difference between the CG and TG ( $\mathrm{p}=0.0624)$ for nonstimulated walking speed. The PCI of nonstimulated walking in CG showed a downward trend that was not significant in either group $(\mathrm{CG}=0.619$ and $\mathrm{TG}=0.072)$. There was no significant difference between CG and TG for nonstimulated PCI (0.13408). Comparison of regression lines for stimulated walking speed with CG showed a highly significant difference $(\mathrm{p}=0.037)$. Regression lines for stimulated PCI were also highly significant $(\mathrm{p}=0.007)$. Results of the analysis of the secondary outcome measures also are presented. Analysis of the results of the primary outcome measures suggest that physiotherapy alone and the combined use of BoNTA and FES with physiotherapy have a beneficial effect on walking speed and PCI. The study has provided us with data that are sufficiently convincing of an additional effect of BoNTA and FES to pursue a larger scale randomized controlled trial and inform the sample size calculation.

FUNCTIONAL ELECTRICAL STIMULATOR COMPEX MOTION. Thierry Keller, ${ }^{1}$ Milos R. Popovic, ${ }^{2}$ Ion P.I. Pappas, ${ }^{2}$ and Pierre-Yves Müller. ${ }^{3}{ }^{1}$ ParaCare, Institute for Rehabilitation and Research, University Hospital Balgrist, Zurich; ${ }^{2}$ Automatic Control Laboratory, Swiss Federal Institute of Technology, Zurich; and ${ }^{3}$ Compex SA, Ecublens, Switzerland

Research groups that work in the field of functional electrical stimulation (FES) are confronted often with the fact that existing and commercially available FES stimulators do not provide sufficient flexibility and cannot be used to perform different FES tasks. The lack of flexibility of the existing commercial systems until now forced various FES research teams to develop their own stimulators. Instead of developing our own stimulator we decided to enhance the existing Compex 2 stimulator, which is already used for various medical and therapeutic applications, by adopting its firmware and by developing a new software that further enhances the stimulator's versatility and capabilities. The stimulator has 4 biphasic current regulated stimulation channels and 2 general purpose analog input channels that can be configured to measure the output voltage of a variety of sensor systems like goniometers, inclinometers, gyroscopes, or electromyographic (EMG) sensors. For real time EMG control of the stimulation patterns an EMG processing algorithm with software stimulation artifact blanking was implemented. With the new firmware and a new graphical user interface software the stimulator can be used now to develop various custom-made neuroprostheses, neurological assessment devices, muscle exercise systems, and experimental setups for physiological studies. The new stimulator, now called Compex Motion, can be programmed to generate any arbitrary stimulation sequence that can be controlled or regulated by various external sensors, sensory systems, or laboratory equipment. By interconnecting multiple Compex Motion stimulators the number of stimulation channels can be expanded to multiples of 4 channels $(8,16,20$, and so on). The stimulation sequences and the control strategies are stored on exchangeable credit card-like memory chip cards. By replacing the chip card the function of the stimulator is changed instantaneously to provide another function or FES treatment. The Compex Motion stimulator is manufactured by the Swiss company Compex SA and is currently undergoing clinical trials.

STIMULATION ARTIFACT REMOVAL ALGORITHM FOR REAL-TIME SURFACE ELECTROMYOGRAPHY APPLICATIONS. Thierry Keller ${ }^{1}$ and Milos R. Popovic. ${ }^{2}{ }^{1}$ ParaCare, Institute for Rehabilitation and Research, University Hos- pital Balgrist, Zurich; and ${ }^{2}$ Automatic Control Laboratory, Swiss Federal Institute of Technology, Zurich, Switzerland

A direct and intuitive method to control a neuroprosthesis for grasping is to use surface electromyography (SEMG) activity of muscles that subjects can voluntarily control; e.g., in the case of C5 or C6 spinal cord injured subjects the deltoid muscles. The measured voluntary SEMG activity in such applications is contaminated with stimulation artifacts (SAs) that are much higher in amplitude compared to raw SEMG signals. Hence, to be able to use SEMG signals for control purposes one has to remove the SA from the measured SEMG signal. In closed-loop applications the SA can produce a positive feedback, which further stresses the importance of removing the SA from the measured signal in closed-loop SEMG control applications. Well-established SA removal techniques are artifact blanking or filtering methods. Realtime SA blanking methods, either hardware built sample-hold circuits or software blanking routines in digitally processed SEMG signals, lose all EMG information during the blanking period. Especially with constant current stimulators, which have very high output impedance, the electrode-tissue impedance can cause an SA of several milliseconds. Most of the SEMG SA filtering techniques are not viable in case of constant current stimulation systems with surface stimulation electrodes since the long lasting SA tail overlaps in frequency and time domain with the voluntary SEMG activity. A new method that encounters the randomness and stationarity of voluntarily generated EMG is presented. An ensemble averaged SA with exponential forgetting was subtracted from the recorded SEMG and an almost artifact free SEMG signal was obtained. Measurements with multichannel stimulation patterns with changing stimulation amplitudes and pulse widths similar to those used in neuroprostheses for grasping showed fast convergence of the algorithm and a good quality of the residual SEMG. The algorithm was less sensitive to changes in the stimulation amplitude than to changes in the pulse width. The method can be implemented in real-time applications and requires a low computational power.

INITIAL RESULTS FROM TWO TRIALS OF AN IMPLANTABLE TWO CHANNEL DROP FOOT STIMULATOR. Laurence Kenney ${ }^{1}$ Hermie Hermens, ${ }^{1}$ Rik Buschman, ${ }^{1,2}$ Gerrit Bultstra, ${ }^{3}$ Paul Taylor, ${ }^{4}$ and Geraldine Mann. ${ }^{4}$ Roessingh Research and Development bv; ${ }^{2}$ Twente Institute for Neuromodulation; ${ }^{3}$ University of Twente, Enschede, The Netherlands; and ${ }^{4}$ Salisbury District Hospital, Salisbury, U.K.

This article describes the early clinical results from 2 parallel trials of a new implantable drop foot stimulator. The stimulator consists of an externally worn transmitter, which is inductively coupled to an implanted receiver unit located in the lower leg, lateral and distal to the knee. The receiver is connected via cables to electrodes located under the epineurium of the deep and the superficial peroneal nerves. Stimulation is triggered by detection of heel lift and terminated at heel strike in a manner similar to other surface mounted systems. The location of the electrodes on the deep and superficial peroneal nerves allows for a degree of selectivity over the resultant moment and movement at the ankle joint that is not possible with surface stimulation of the common peroneal nerve. The first implant took place in The Netherlands in July 2000. Since then, a further 3 implants have taken place, 1 in The Netherlands and 2 in the U.K. The subjects are all cardiovascular accident patients, at least 3 years poststroke and between 31 and 48 years old. The U.K. and Dutch trials both used a single case study approach. Baseline data on walking speed and endurance were gathered on at least 3 separate occasions, both without and with the normal walking aid (if any). The U.K. group also measured physiological cost index data. Prior to the implant operation nerve conduction measures were taken to check the integrity of the deep and superficial peroneal nerves. Following implantation the nerve conduction measures were repeated and walking speed, endurance, and isometric torque measures were 
taken over a period of 12 weeks. The early results indicate that the system is safe to use, well liked by the patients, provides selectivity over moments at the ankle joint, and increases both walking speed and endurance. Three of the 4 systems continue to function without any problems. An intermittent fault has been found with the second system to be implanted, the cause of which is currently under investigation. The results will be presented in the full article.

FUNCTIONAL ELECTRICAL STIMULATION TRAINING OF DENERVATED MUSCLES IN HUMANS. H. Kern, ${ }^{1}$ Ch. Hofer ${ }^{1}$ M. Strohhofer, ${ }^{1}$ and W. Mayr. ${ }^{2}{ }^{1}$ Department of Physical Medicine, Wilhelminenspital; and ${ }^{2}$ Department of Biomedical Engineering and Physics, University of Vienna, Vienna, Austria

We examined the possibility of training denervated human muscles by means of functional electrical stimulation (FES) in complete denervated paraplegics. Patients with complete conus cauda lesion (Frankel A) were examined to see if by using appropriate electrical stimulation muscular degeneration could be prevented and already degenerated muscles could be improved to an extent that tetanic contractions and functional movements could be triggered using FES. These patients started training with electrical stimulation 2 years after the lesion. Tetanic muscle contractions with knee extension moments of 16.5 to $38 \mathrm{Nm}$ could be obtained after 16 to 26 months. Muscle training was begun in the sitting position with single twitches (biphasic rectangular pulse, average pulse width $120 \mathrm{~ms}$ ) followed by tetanic contraction cycles (on 1-2 s, off 2-4 s) initially without weight until nearly complete knee extension was achieved and later with weight cuffs $(0.5-1 \mathrm{~kg}$ on the ankle, $6-12$ repetitions, 6 series, once and later twice daily). Endurance training was performed by additional stimulation with 5 to $8 \mathrm{~Hz}$ with $10 \mathrm{~s}$ contraction time and $1 \mathrm{~s}$ break later followed by $5 \mathrm{~s}$ contraction time and $1 \mathrm{~s}$ break (pulse duration $60-70 \mathrm{~ms}$ ). Improvement in muscle force $(53-73 \%)$ and muscle cross section area $(22-58 \%)$ within 1 year of tetanic muscle training was documented by means of torque measurement and computer tomography respectively. As knee stabilization in the standing position requires relatively low moment and standing up is supported by the upper body, standing training can be started early on. In our patients FES started 1 to 2 years after lesion (complete conus cauda). Standing training could be carried out 9 to 12 months after commencement of FES.

FATIGUE PROCESS OF TYPE 1 AND TYPE 2 MUSCLE FIBERS. Katarina Kerševan, Vojko Valenčlč, and Nataša Knez. University of Ljubljana, Faculty of Electrical Engineering, Ljubljana, Slovenia

The skeletal muscle fatigue process was examined in biceps brachii and tibialis anterior muscles in 9 male subjects age 21 to 24 years. Measurements were carried out under isometric conditions. Pulse trains $(0.1 \mathrm{~ms})$ of different stimulation frequencies and voltage were used for $10 \mathrm{~s}$ electrical stimulation and a noninvasive tensiomyographic method was used for detection of the muscle responses to electrical stimulation. Using pulse trains of different frequencies enabled separate observation of the fatigue and tetanization process for both muscle fiber types. Type I (fatigue resistant) muscle fibers generated and retained force throughout the $10 \mathrm{~s}$ electrical stimulation. The fatigue process was observed at a stimulation frequency of $2 \mathrm{~Hz}$ but the Type I muscle fiber fatigue did not occur due to their fatigue resistant character. Complete tetanus of Type I muscle fibers was noticed at stimulation frequency of 4 to $6 \mathrm{~Hz}$. The fatigue process of Type II (fast fatigue) muscle fibers ended before the electrical stimulation was over and was noticed at stimulation frequencies of 7.5 to $9 \mathrm{~Hz}$. Complete tetanus of Type II muscle fibers was noticed at stimulation frequencies higher than $18 \mathrm{~Hz}$. Observation of Type II muscle fibers also enables more efficient treatment and observation of patho- logical changes in dystrophic, atrophic, and denervated patients as well as helps professional athletes and their trainers to improve their training technique.

A COMBINED STIMULATION AND TELEMETRIC MONITORING SYSTEM: CURRENT DEVELOPMENT FOR BIOMECHANICAL HEARTS. P. Klapproth ${ }^{1}$ N.W. Guldner, J. Otten ${ }^{2}$ P. Maczinowski, ${ }^{2}$ B. Nestler, ${ }^{2}$ M. Ryschka, ${ }^{2}$ T. Büter, ${ }^{2}$ and R. Schirmer. ${ }^{2}{ }^{1}$ Clinic of Cardiac Surgery, Medical University of Lübeck; and ${ }^{2}$ University of Applied Science, Lübeck, Germany

Current myostimulator systems allow via nearfield telemetric programming the adjustment of the electrical stimulation pattern applied to muscle driving cardiac assist devices. Thus, the stimulation parameter could be adapted to a user defined stimulation protocol varying the pulse amplitude, the pulse width, the number of pulses per burst, the interpulse interval (constant, incremental, and decremental), the number of bursts per minute respiratory heart beat in R-wave-triggered mode, the R-wave-distance of the burst, and so on. Although the available stimulation systems allow a precise setting of the mentioned stimulation parameter, some features for experimental and later clinical purposes are desirable. This includes a variable day and night rhythm of the burst delivery, a late diastolic assist in a counterpulsator system as well as the exact control about the placement of each single pulse within a burst in order to use the doublet-effect resulting in sufficient muscle contractions with less pulses. In addition to the stimulation task, a new design could include a measurement unit that evaluates the electrocardiogram (ECG) but also the blood pressure with a newly developed microelectromechanical system (MEMS)based hybrid pressure sensor. In order to forward the evaluated data, the measurement unit should be coupled with a telemetric system, where the data might brought to a receiving computer. Thus, the data are available for external access. Our myostimulator prototype consists of 2 microcontrollers, 1 for the myostimulator and 1 for the measurement unit. An R-wave detection unit gives the trigger signal for the synchronization of the heart beat and the stimulation burst. With an 8 channel digital analog converter and current amplifier, a software controlled stimulation pattern is applicable. The second microcontroller, which is almost in sleep mode, could become activated cyclic. With its 8 channel, 12 bit analog digital converter and 316 bit timer, the ECG, the impedance of the stimulation electrodes, the battery voltage, as well as up to 3 pressure sensors are independently evaluable. Via the integrated telemetry these data could be transmitted to an external computer and a new stimulation protocol as well as a new software for the controller could be received. Further features like intrinsic monitoring of the heart frequency and the blood pressure should become integrated.

CONDITIONING PROTOCOL AND STIMULATION DEVICE OF THE VIENNA SKELETAL MUSCLE VENTRICLE PROJECT. H. Lanmüller, ${ }^{1}$ U. Windberger,${ }^{1}$ S. Sauermann,${ }^{1}$ M Rab, ${ }^{2,3}$ E. Unger, ${ }^{1}$ and W. Girsch. ${ }^{4}{ }^{1}$ Department of Biomedical Engineering and Physics, ${ }^{2}$ Department of Biomedical Investigation, ${ }^{3}$ Department for Plastic and Reconstructive Surgery, University of Vienna; and ${ }^{4}$ Orthopaedic Hospital Speising, Vienna, Austria

The Vienna skeletal muscle ventricle (SMV) is designed to perform counterpulsation parallel to the descending aorta. The neoventricle is created by enlarging a segment of a thoracic aorta with pericardium, both from a donor, driven from the latissimus dorsi muscle (LDM). This configuration has been proved in acute experiments and now in a ongoing chronic animal experiment. In the chronic study the LDM is conditioned in situ before construction of the SMV. Conditioning is started 1 week after electrode implantation with 30 contractions per minute. The number of pulses per contraction is incremented biweekly from 2 pulses up 
to 5 pulses. Conditioning is completed after 8 weeks and results in a completely transformed muscle with slow-twitching Type I fibres. The connective tissue increases from $17.7 \%$ to $22.7 \%$. At the beginning of the study an implantable nerve stimulator was applied in 4 adult sheep. This device was developed at our own department, powered by a lithium thionyl chloride battery and hermetically sealed in a titanium case. To achieve a reduction in costs this device was modified into a semi-implantable version. In this version only the electrode leads are implanted chronically. Three sensing leads and 4 epineural stimulation leads are connected to a single skin button with an integrated connector. The external ECG triggered pulse generator is fixed on the animal by an elastic belt, and its functionality is equal to the implantable version. Up to now the semi-implantable stimulator was used in 4 adult goats. An implantation period of 4, 7, and 5+ (now in operation) months was reached without complications. An infection because of the percutaneous link appeared in 1 goat after 7 months. Overall, this resolution turned out as a well usable and cost-saving device for animal experiments.

DEVICES FOR NONINVASIVE TRANSCRANIAL ELECTROSTIMULATION OF THE BRAIN ENDORPHINERGIC SYSTEM: APPLICATION FOR IMPROVEMENT OF HUMAN PSYCHOPHYSIOLOGICAL STATUS. V.P. Lebedev ${ }^{1}$ A.V. Malygin, ${ }^{1}$ A.V. Kovalevski, ${ }^{1}$ V.N. Sisoev, ${ }^{2}$ S.P. Kropotov, ${ }^{2}$ E.M. Krupitski, ${ }^{3}$ L.I. Gerasimova, ${ }^{4}$ and D.V. Glukhov. ${ }^{5}{ }^{1}$ Pavlov Institute of Physiology, ${ }^{2}$ Military Medical Academy, ${ }^{3}$ Regional Narcological Dispensary, Saint Petersburg; ${ }^{4}$ Sklifasovsky Research Institute of Emergency; and ${ }^{5}$ Medical Center of Extreme Situations, Moscow, Russia

It is well known that deficit of endorphins play an important role in disturbances of human psychophysiological status. Previously we revealed that brain endorphinergic structures have a quasiresonance characteristic. On the basis of these data a method of activation of the brain endorphinergic structures by means of noninvasive and rather selective transcranial electrostimulation (TES) as a kind of functional electrical stimulation (FES) was elaborated. The aim of this article is the description of TES devices and effects of their applications for reduction of psychophysiological disturbances elicited by stress of different intensities and some other factors. New models of TES devices (TRANSAIR) were developed for in and outdoor use. Special adjustment of output rectangular current impulses was done to reduce the unpleasant skin sensations. To increase the efficacy of TES the frequency modulation according normal distribution in the limits of the quasiresonance characteristics was put into operation. The blind and placebo-controlled (passive and active placebo) study was produced to estimate the TES effects on stress events and accompanying psychophysiological and autonomic disturbances of different intensity in following groups of volunteers and patients: everyday stress and fatigue, stress in regular military service and in field conditions, stress in the relatives of the lost in mass disasters, posttraumatic stress (thermal burns), and affective disorders in postabstinence periods. Some subjective verbal and nonverbal tests and objective tests (including heart rate variability) were used for estimation of initial level of psychophysiological status and its changes after TES sessions. It was demonstrated that fatigue, stress, and other accompanying psychophysiological disturbances were significantly improved or abolished after 2 to 5 TES sessions. The TES effects were more pronounced in cases of heavier disturbances. In conclusion, activation of the brain endorphinergic structures by TES is the effective homeostatic method of FES that sufficiantly improves the quality of life.

RIGOROUS GREEN'S FUNCTION FORMULATION FOR INDUCED TRANSMEMBRANE POTENTIAL ALONG THREE-DIMENSIONAL INFINITE CYLINDRICAL CELL. L.M. Livshitz, ${ }^{1}$ P.D. Einziger, ${ }^{2}$ and J. Mizrahi. ${ }^{1}{ }^{1}$ Departments of
Biomedical and ${ }^{2}$ Electrical Engineering, Technion, Israel Institute of Technology, Haifa, Israel

The quasistatic electromagnetic field interaction with 3-D infinite cylindrical cell is investigated for both intra- and extracellular current point source excitations, IPS and EPS, respectively. The induced transmembrane potential, expressed conventionally via Green's function, may be expanded alternatively into a faster converging representation using a complex contour integration, consisting of an infinite discrete set of exponentially decaying oscillating modes (corresponding to complex eigenvalues) and a continuous source-mode convolution integral. It is insensitive to large variations of the internal/external conductivity ratio as long as the membrane/external (or membrane/internal) conductivity ratio is maintained very low. The dominant contribution for both the IPS and EPS problems are obtained in simple closed-form expressions, including well-documented special functions. In the IPS case the dominant modal contribution (of order 0), an exact solution of the well-known cable equation, is explicitly and analytically corrected by the imaginary part of its eigenvalue and the source-mode convolution contribution, both of the order of the membrane/external conductivity ratio. The limit where the external conductivity approaches infinity is well documented in the literature. In this limit the convolution integral contribution vanishes and complex eigenvalues become real. In the EPS case the dominant contribution is expressed as a source-mode convolution integral. However, for a long EPS distance ( $\geq 50$ fiber diameters) the mode (of order 1) involved in the convolution is not a solution of the cable equation. Only for shorter EPS distance $(\approx 5$ fiber diameters) should the cable equation solution (i.e., mode of order 0 ) be included in addition to the dominant mode (of order 1). For on-membrane EPS location ( $\approx 1$ fiber diameter) additional modes should be included as well. In view of our EPS result, we suggest that the cable equation analysis and modeling already contained in the literature and related to functional electrical stimulation for EPS problems critically should be reviewed and corrected.

\section{MUSCLE ACTIVITY DURING NORMAL WALKING AND ITS RELEVANCE FOR FUNCTIONAL ELECTRICAL STIMULATION APPLICATIONS. Sabine Mangold, ${ }^{1}{ }^{\text {Thierry }}$ Keller, ${ }^{2}$ and Milos R. Popovic. ${ }^{1}{ }^{1}$ Automatic Control Laboratory, Swiss Federal Institute of Technology, ETH Zürich, Zürich; and ${ }^{2}$ ParaCare, University Hospital Balgrist, Zurich, Switzerland}

Exact knowledge of the temporal pattern of muscle activity during walking is essential for the improvement of locomotion that is generated or supported by functional electrical stimulation (FES). Thus, the objective of this study was to give an overview of the activity of functional muscle groups that control the hip, knee, ankle, and subtalar/transverse tarsal joints' movements during normal walking. In the literature more than 20 muscles were identified as the muscles that actuate these joints during walking on a plain surface. The mean electromyographic profiles of these muscles, which were described in the literature, were compared by extracting 3 levels of muscle activity: $25 \%, 50 \%$, and $75 \%$ of the maximum activity. Agonistic muscles were identified and analyzed with respect to their function and estimated activity level during the single gait phase cycle. We believe that this information is essential in identifying which muscles and muscle groups have to be stimulated, to which extent, and in which sequence to facilitate true dynamic walking. At the moment the existing FES systems that support or facilitate walking generate gait patterns that look like set of static leg positions with abrupt and jerky transitions between these static positions. Since such static walking patterns are slow and physically demanding it is essential that the new generations of FES systems for walking facilitate gait cycles that resemble and mimic able bodied subjects' dynamics during walking. Also, this study would help us assess if the FES technology with surface stimulation electrodes can be used to generate the dynamic walking and what are the design requirements for such a system. 
A PILOT STUDY TO INFORM SAMPLE-SIZE CALCULATIONS FOR AN INVESTIGATION INTO THE EFFECTS OF ELECTRICAL STIMULATION ON RECOVERY OF HAND SENSATION AND FUNCTION IN STROKE PATIENTS. G.E. Mann, ${ }^{1}$ J.H. Burridge, ${ }^{2}$ L.J. Malone, ${ }^{1}$ and P.N. Taylor. ${ }^{1} 1$ Department of Medical Physics and Biomedical Engineering, Salisbury District Hospital, Salisbury, Wiltshire; and ${ }^{2}$ Department of Health Sciences and Rehabilitation, University of Southampton, Southampton, U.K.

Recovery of motor function rather than associated sensory deficits is the main focus of rehabilitation therapy for the upper limb following stroke in the United Kingdom. Sensory deficits are known to have an adverse effect on functional outcome, although their severity is not necessarily related to degree of functional loss. Studies that have included assessment of sensation have proved inconclusive. A retrospective study of stroke patients with restricted hand function and sensation, referred to the authors for electrical stimulation treatment, showed improvement in sensation in the hemiplegic hand in 7 out of 11 patients tested and measured using static 2 point discrimination of 24 areas of the hand. It is recognized that this previous study had design and methodological limitations. The purpose of this study was to inform sample-size calculations for a full randomized controlled trial to investigate the effects of electrical stimulation on recovery of hand sensation and function in stroke patients. Thirty subjects, within 1 year following a first stroke, are randomized into equal treatment and control groups. The treatment group receives electrical stimulation of elbow and wrist extensors and the control group elbow and wrist extension exercises. Assessments of upper limb function use the Action Research Arm Test and the Jebsen Taylor Hand Function Test. Hand sensation is assessed using Static Two Point Discrimination. Assessments are conducted pretreatment (Week 0) midtreatment (Week 6), and posttreatment (Week 12) and 12 weeks posttreatment (Week 24). Fifteen subjects ( 9 female and 6 male) have so far been recruited to the study, between 2 months and 1 year after stroke. Of these 1 has completed the study and 6 others have completed the treatment period only.

EUROPEAN UNION PROJECT RISE: USE OF ELECTRICAL STIMULATION TO RESTORE STANDING IN PARAPLEGICS WITH LONG-TERM DENERVATED DEGENERATED MUSCLES. W. Mayr, ${ }^{1} \mathrm{H}$. Kern, ${ }^{2}$ C. Hofer ${ }^{2}{ }^{\mathrm{J}}$. Jarvis, ${ }^{3}$ and S. Salmons. ${ }^{3}{ }^{1}$ Department of Biomedical Engineering and Physics, University of Vienna; ${ }^{2}$ Department of Physical Medicine, Wilhelminenspital, Vienna, Austria; and ${ }^{3}$ Department of Human Anatomy and Cell Biology, University of Liverpool, Liverpool, U.K.

A novel clinical rehabilitation method for patients who have permanent and nonrecoverable muscle denervation in the legs will be developed. The technique based on functional electrical stimulation (FES) will restore muscle tissue, muscle function, and the ability to rise in these severely disabled patients. Such an improvement in mobility will confer a substantial reduction in the risk and severity of bed sores and other secondary medical problems, resulting in less frequent hospitalization and a reduced burden on public health services, as well as improvements in the patient's quality of life. Soundly based and comprehensive guidelines will be developed for the safe and effective clinical use of FES in this challenging new area, and all the associated technology will be delivered in a near-market form. The work is organized in 3 experimental and 2 technical work packages. The first phase, to be conducted in rabbits, is designed to determine optimal electrical parameters and the amount and intensity of FES training needed to achieve a functionally useful muscle in the shortest possible time. Stimulation will be applied to rabbit tibialis anterior muscles via implantable stimulators and epimysial electrodes and the results evaluated with a comprehensive body of physiological, histochemical, histological, and biochemical tech- niques. This part of the study will establish a safe envelope of stimulation parameters that do not pose a risk of damage to the muscle. Further experiments will be conducted to determine the stimulation conditions needed to maintain the trained status after it has been achieved. Use of the rabbit model will enable a large number of variables to be explored in a short time and will narrow the focus of the study in terms of the intensity, duration, and regularity of the FES sessions needed. In the next phase of the study we will begin the process of transferring the findings from the rabbit to the pig, whose musculature offers a closer approximation to human muscles in terms of muscle size and contractile characteristics. Again the muscles will be characterized by physiological, histochemical, histological, and biochemical measurement techniques. In the last phase of the project the results of the animal studies will be applied clinically. The training period needed to mobilize the patient could last for up to 2 years, depending on the initial status of the muscles. The associated technology will be under development throughout the project, guided by the results of the animal experiments and initial clinical trials. The main devices will be a portable stimulator, electrodes, control elements, and training aids for use by the patient, and measurement and test equipment for the physician in the out-patient clinic.

FUNCTIONAL ELECTRICAL STIMULATION AS A COUNTERMEASURE AGAINST MUSCULAR ATROPHY IN LONG-TERM SPACE FLIGHTS. W. Mayr, ${ }^{1}$ G. Freilinger, ${ }^{1}$ D. Rafolt,${ }^{1}$ M. Bijak, ${ }^{1}$ W. Girsch, ${ }^{1}$ C. Hofer, ${ }^{1}$ H. Lanmueller, ${ }^{1}$ M. Reichel, ${ }^{1}$ S. Sauermann, ${ }^{1}$ C. Schmutterer,${ }^{1}$ E. Unger,${ }^{1}$ V. Gratchev, ${ }^{2}$ Y. Koryak, ${ }^{2}$ B. Shenkman, ${ }^{2}$ I. Belozerova, ${ }^{2}$ I. Kozlovskaya, ${ }^{2}$ and A. Gregoriev. ${ }^{2}{ }^{1}$ Department of Biomedical Engineering and Physics, University of Vienna, Vienna, Austria; and ${ }^{2}$ Institute for Biomedical Problems, Moscow, Russia

Long-term flights in microgravity cause atrophy and morphological changes of skeletal muscles. Training with mechanical devices is insufficient regarding time to exercise and space for devices. The objective of the project MYOSTIM is to develop a training method based on functional electrical stimulation (FES) to preserve muscle mass and fiber composition with minimal impairment to the cosmonaut. For a pilot experiment on board the MIR station suitable 8 channel FES equipment was developed. It consists of electrode trousers that carry surface electrodes and cables, 2 interconnected 4 channel stimulators, and a laptop PC for stimulator programming and processing compliance data. An automatic extensive training of 4 muscle groups of the lower extremities is performed for $6 \mathrm{~h}$ per day with $1 \mathrm{~s}$ on- $2 \mathrm{~s}$ off tetanic contractions at $20 \%$ to $30 \%$ of maximum tetanic muscle force. Synchronous activation of antagonists of thigh and lower leg prevents uncoordinated movements. The first successful test on board MIR was performed by 2 cosmonauts between December 1998 and February 1999 and March 19999 and August 1999 respectively.

LEG POWERED PARAPLEGIC CYCLING SYSTEM USING SURFACE FUNCTIONAL ELECTRICAL STIMULATION. T.A. Perkins, ${ }^{1}$ N. de N. Donaldson, ${ }^{1}$ R. Fitzwater, ${ }^{2}$ G.F. Phillips, ${ }^{2}$ and D.E. Wood. ${ }^{3}$ University College, London; ${ }^{2}$ Royal National Orthopaedic Hospital, Stanmore; and ${ }^{3}$ Salisbury Hospital, Salisbury, U.K.

We aimed to enable our partial lesion T11/12 male paraplegic subject to achieve outdoor leg-powered cycle rides that would give exercise, health benefits, and mobility using surface functional electrical stimulation (FES). We hoped that improved function would encourage greater effort, particularly as more intense exercise reportedly has given some recovery in bone density as well as the other health benefits expected from moderate exercise 
levels. Commercially available recumbent tricycles were obtained for their inherent stability and low seating pressure. Switches were attached to the handlebars for starting and stopping the cycling program. A 7 bit shaft encoder was connected to the cranks. Purpose built platforms were attached to the pedals to help secure the feet. A throttle potentiometer adjusted overall stimulation strength (and hence pedaling speed). A resistance trainer stand was provided so that the tricycle was turned into a static ergometer for indoor cycle exercise at home. We used an 8 channel surface stimulation controller, giving pulses of up to $500 \mu \mathrm{s}$ and $150 \mathrm{~mA}$. Quadriceps, hamstring, and gluteal muscles on both sides were activated, with gastrocnemius and tibialis anterior on the more paralyzed left side. Stimulation frequency was $20 \mathrm{~Hz}$. Stimulation control was derived from the crank angle shaft encoder, having set start and stop angles for each muscle group. A $150 \mathrm{~ms}$ time-advance compensated for muscle response delay and accommodated changes in cadence from about 25 to $55 \mathrm{rpm}$. Over the first 16 months, the patient averaged 21 min of daily FES training with a simpler system. He has exercised for a further year with the system described here. At 20 months, bone density in his left tibial tuberosity had increased by $44 \pm 2 \%$. With this new system, our subject has cycled $12 \mathrm{~km}$ at a time on near level ground outdoors. We have clearly established a practicable system for FES leg cycling. This may be a valuable function following spinal cord injury, giving considerable health benefits. We are extending currently the use of the system to complete lesion paraplegics.

SELF-TUNING REGULATION OF MUSCLE GENERATED MOMENT INDUCED BY ELECTRICAL STIMULATION. Matija Ponikvar and Marko Munih. Faculty of Electrical Engineering, University of Ljubljana, Ljubljana, Slovenija

This article presents the development, implementation, and experimental evaluation of an adaptive feedback system for control of the muscle generated moment. The control system, which utilizes self-tuning regulator (STR) and real-time estimation of local muscle model parameters, triggered the stimulator and muscle contractions. Design of such a moment controller represents the base stage leading toward the construction of a position controller. In order to simplify in vivo experiments with functional electrical stimulation, we focused only on gastrosoleus muscles under isometric conditions. The stimulated subject stood in a mechanical rotating frame (MRF), which prevented rotation of knee, hip, and lumbosacral joints. The MRF is locked, meaning that also the ankle joint is stiff. The moment in the ankle as output of the standing human was measured separately for left and right foot with 2 force plates. The STR was then used to automatize the muscle model identification and the calculation of controller parameters. The fidelity of muscle models was estimated on-line with the least square recursive method, which enables the implementation of adaptive control. Adaptive control of electrically stimulated muscle can in real time embrace the changes caused by time-varying muscle behavior such as fatigue. On-line identification does not require any advance identification procedures and stimulation. The control law was based on the pole placement design that gives desired closed-loop poles. The STR was realized with the program Matlab Simulink by using program blocks for identification part, controller, force plates, and computer controlled electrical stimulator. The linear controller operation was then tested in 3 activation regions between stimulation threshold and saturation to exclude nonlinear activation effects. For all 3 activation regions were utilized different controller configurations with advance adjustment of tuning parameters that indirectly reflect the desired closed-loop transfer function. Sinusoidally shaped moment trajectories included oscillation frequencies between 0.1 and $1.5 \mathrm{~Hz}$. The muscles were stimulated with controlled repetition pulses at $20 \mathrm{~Hz}$. The controller tracking was demonstrated to be satisfactory. However, due to simple adaptive constitution it was not robust enough to attenuate well larger disturbances.

RESTITUTION OF REACHING AND GRASPING PROMOTED BY FUNCTIONAL ELECTRICAL THERAPY. M.B. Popovic, ${ }^{1,2}$ T. Sinkjær, ${ }^{1}$ D.B. Popovic, ${ }^{1}$ A. Stefanovic, ${ }^{3}$ and L. Schwirtlich. ${ }^{3}{ }^{1}$ Center for Sensory Motor Interaction, Aalborg University, Aalborg, Denmark; ${ }^{2}$ Institute for Medical Research; and ${ }^{3}$ Institute for Rehabilitation "Dr Miroslav Zotovic," Belgrade, Yugoslavia

We hypothesized that a significant recovery of functioning is promoted by functional electrical therapy (FET) shortly after stroke. The study included 16 subacute stroke subjects, divided into 2 groups: a low functioning group (LFG), and a high functioning group (HFG) based on their ability to control wrist and fingers. The subjects were randomly associated with the FET and control groups. The FET was applied with the Belgrade grasping system (BGS). The BGS was applied during 30 min daily sessions during 3 consecutive weeks. The therapy consisted of reaching, grasping, and trying to functionally use the typical daily necessities (e.g., pen, telephone receiver, can) during the sessions. The blinded outcome measures were the upper-extremity function test (UEFT; 11 tasks) and coordination between the shoulder and elbow (the ability to draw on the digitizing board) before and after the therapy. The change in performance of the FET group was significant. The coordination and UEFT were improved for more than $55 \%$ in the HFG being subjected to FET $(23 \%$ in the controls). The coordination and UEFT changed to $68 \%$ in the LFG subjected to FET ( $12 \%$ in the controls), yet the change is not good enough to provide daily functioning. This may be because the effects deteriorate at lingering time because of disuse. The follow-up will continue for the next 18 months. The therapeutic effects of the FET could be associated with numerous mechanisms. First, the electrical stimulation is generating very strong central input that is combined with the volitional commands. This sensory input is likely to become integrated into the newly developed sensory-motor scheme being responsible for the improved functioning. Second, the electrical stimulation is strengthening the muscles that are weak due to disuse atrophy.

FUNCTIONAL ELECTRICAL STIMULATION INDUCED SURFACE MUSCLE STIFFNESS CAPTURED BY COMPUTER CONTROLLED TONOMETRY. D. Rafolt, E. Gallasch, M. Bijak, H. Lanmüller, S. Sauermann, M. Fend, and W. Mayr. Department of Biomedical Engineering and Physics, University of Vienna, Vienna, Austria

The surface stiffness produced over a muscle belly is a function of passive viscoelastic and active contractile tissue properties. If surface stiffness is sensed by local indentation (in vivo) further than the skin interface, tissue geometrical and bone architectural factors have to be considered. To evaluate functional electrical stimulation induced tissue mechanical changes in calf muscles $(\mathrm{m}$. triceps surae, $\mathrm{m}$. tibialis) a new computer controlled tonometric test system was set up. The tonometric system is built around an isometric ankle dynamometer that serves as a mechanical reference for the indentator and as a measurement device for the contraction force (plantar flexion) and the skin myoelectric activity. The skin surface indentator is driven by a force controlled actuator (based on a galvanometer scanner with an embedded deflection sensor) to capture the local surface stiffness as well as dynamic force responses to single stimulation pulses and burst stimulation patterns. Special real time software was developed to synthesize the test stimulation patterns, to control the indentation forces, and to capture the measurement data. This software also includes computer display markers to study stiffness changes in response to voluntary dynamometer contractions. The analysis software consists of routines for data visualization and parameter estimation such as the force/stiffness relationship. Viscoelastic phantoms are used to calibrate the indentation mechanics. 
TWITCH SUMMATION WITH DOUBLE STIMULATION. A. Rainoldi, ${ }^{1,2}$ W.K. Durfee ${ }^{3}$, and R. Merletti. ${ }^{2}$ Centro di Bioingegneria, Dip. di Elettronica, Politecnico di Torino, Turin; ${ }^{2}$ Department of Physical Medicine and Rehabilitation, Università di Tor Vergata e Fondazione Don Gnocchi, Rome, Italy; and ${ }^{3} \mathrm{De}-$ partment of Mechanical Engineering, University of Minnesota, Minneapolis, Minnesota, U.S.A.

The use of doublets in functional electrical stimulation applications is a well-known technique aimed to increase force with a concomitant reduction of muscular fatigue. This work evaluated the effect of doublets on both force and electromyographic (EMG) signals (biceps brachii muscle) and assessed the repeatability of results in different days. Five healthy subjects (27.8 \pm 3.8 years) were involved in this experiment. The protocol consisted of 1 session with 5 different trials in the following order: 25 singlets (single $50 \mu$ s rectangular pulse), 200 doublets ( 2 equal rectangular pulses separated in time by the pulse interval [PI]) with PI in the ranges 1 to $5 \mathrm{~ms}, 1$ to $20 \mathrm{~ms}, 1$ to $100 \mathrm{~ms}$ and, finally, 25 singlets. Every stimulus (either singlet or doublet) was generated once a second and trials were separated by $10 \mathrm{~min}$ of rest. The whole session was repeated in 3 different days. The force twitches generated by these impulse trains were recorded by means of an isometric mechanical brace with resonance frequencies outside the force signal bandwidth. The EMG signals were recorded by means of a 4 bar electrode array placed distally on the muscle between the innervation zone and the tendon. The studied variables were peak of force (PF), time to reach the peak (TTP), half relaxation time, twitch area (TA), conduction velocity $(\mathrm{CV})$, and the correlation coefficient between 2 double differential signals. The day to day repeatability was poor; therefore, by means of a multicriteria method the best session for each subject was selected. The absolute refractory period (ARP) average value was $2.2 \pm 0.1 \mathrm{~ms}$. The average maximal ratio between PF obtained with doublets and with singlets was $2.79 \pm 0.93$ (2.12-3.96), quite constant within subjects (the average standard deviation between days was 0.28 ), and was obtained for PI $=3.5 \pm 0.1 \mathrm{~ms}$. The TTP was greater in double response with respect to singlets for PI > ARP. The minimum values of TA correspond to the singlets while the maximum value was observed for PI just larger than ARP and remained constant for ARP $<$ PI $<30 \mathrm{~ms}$. The 2 stimuli in each doublet generated $2 \mathrm{M}$-waves. The second wave had CV larger than the first for $23<$ PI $<100 \mathrm{~ms}$. The difference was significant $(\mathrm{p}<0.01)$ in 4 subjects out of 5 .

SIMULATION OF THE THREE-DIMENSIONAL ELECTRIC FIELD IN THE COURSE OF FUNCTIONAL ELECTRICAL STIMULATION. M. Reichel, ${ }^{1}$ T. Breyer, ${ }^{1}$ W. Mayr, ${ }^{1}$ and F. Rattay. ${ }^{2}{ }^{1}$ Department of Biomedical Engineering and Physics, University of Vienna; and ${ }^{2}$ Department of Analysis and Technical Mathematics, Vienna University of Technology, Vienna, Austria

Optimization of stimulation parameters as well as shape and positioning of electrodes are important questions in functional electrical stimulation (FES) of paraplegic patients. For that reason a MATLAB tool, named FES-FIELD, modeling the threedimensional electric field in the human body, has been developed to calculated the electric field in a region of interest. The simulation tool provides a graphic user interface. In case of denervation of the lower extremities an important target muscle is the $\mathrm{m}$. quadriceps femoris. The electrical potential distribution along its fibers is representative for its functional activation. For this special application the human thigh stimulated by skin electrodes has been modeled. The simulation process has been done in five steps:

(1) Reading the geometric information of the thigh from 50 computed tomographic slices (256 by 256 pixels);

(2) Segmentation in tissue types like fat, muscle, bone, and so on by pixel value and definition of each conductivity;

(3) Selection of electrode geometry and positioning;
(4) Calculating the electric field iteratively by solving the system of approximately 1,500,000 linear equations; and

(5) Visualization of the solution by equipotential lines in either cross or length sections of the thigh.

The simulation requires 5 to $6 \mathrm{~h}$ times for approximately 6,000 iterations computed with a standard PC ( $800 \mathrm{MHz}$ CPU, $512 \mathrm{MB}$ RAM). The graphic information and the electrical solution can be exported to binary files for further investigations like calculation of the activating function of representative fibers in several muscle regions.

BIPHASIC STIMULATION: AN ALTERNATIVE APPROACH TO MINIMIZE THE STIMULUS ARTIFACT FOR DIAGNOSTIC AND FOR CONTROL APPLICATIONS. M. Reichel,${ }^{1}$ M. Bijak,${ }^{1}$ W. Mayr ${ }^{1}$ H. Lanmüller, ${ }^{1}$ D. Rafolt,${ }^{1}$ S. Sauermann, ${ }^{1}$ E. Unger ${ }^{1}$ and E. Turkof. ${ }^{2}{ }^{1}$ Department of Biomedical Engineering and Physics and ${ }^{2}$ Department of Plastic and Reconstructive Surgery, University of Vienna, Vienna, Austria

Diagnostic methods, where the electrical response to an electrical stimulus is recorded, provoke charging processes in the electrode and tissue interface. In the recorded signal the stimulation artifact normally strongly dominates in relation to the electrical stimulation response. If the response signal and the artifact overlap, the response signal is lost due to overdrive of the recording amplifier. This especially occurs during Intraoperative motor evoked potentials in brachial plexus surgery and scoliosis surgery, where the stimulation amplitude may reach several hundreds volts: The ratio of the stimulation amplitude and the evoked, efferent motor electroneurogram is in the range of $10^{8}$ together with a short latency ranging between 2 and $4 \mathrm{~ms}$. Similar problems occur during intraoperative localization of chronic facial nerve lesions, where the stimulation site (skull) and the recording site (facial nerve trunk) are especially close to each other, with latency down to $1 \mathrm{~ms}$. Considering the fact that every rising edge of the stimulation impulse leads to a charging current and that every falling edge leads to a discharging current in the capacitive part of the load impedance, the 2 edges of a monophasic stimulation impulse produce a slowly decreasing discharge current through the tissue after the second (falling) edge. In order to minimize the remaining charge at the end of the impulse, we used a biphasic stimulation impulse with asymmetric length of the phases. By adjusting the ratio of the durations of the first and second phase, the remaining discharge current can theoretically be driven toward 0 . Reducing the electrode-tissue interface to an equivalent network of a resistor, the phase duration ratio can be calculated as described in the Methods section. The resistor represents the tissue that is connected serially to a parallel circuit of a capacitor and a resistor, both representing the electrode-skin junction. This ratio depends on the time constant $\tau$ of the equivalent network, which is in the range of 0.5 to $2 \mathrm{~ms}$, and the duration of the first stimulation phase $T_{1}$ that is normally used in the range of 50 to $500 \mu \mathrm{s}$. For example, with a pulse width of the first phase of $50 \mu \mathrm{s}$, the percentage of the second phase $T_{2}$ of the stimulation impulse needs to be $95 \%$ to optimally minimize the stimulus artifact at a time constant of $1 \mathrm{~ms}$ and with a pulse width of $500 \mu \mathrm{s}$; this percentage needs to be $65 \%$. This shows that longer time constants need shorter second phases for optimal compensation and vice versa. The theoretical findings were confirmed by a series of animal and clinical experiments.

MOBILE PERSONAL COMPUTER SYSTEM FOR INTRAOPERATIVE ELECTRONEURODIAGNOSTICS. M Reichel, ${ }^{1}$ M. Bijak,${ }^{1}$ W. Mayr,${ }^{1}$ H. Lanmüller, ${ }^{1}$ D. Rafolt,${ }^{1} \bar{M}$. Rakos, ${ }^{1}$ S. Sauermann, ${ }^{1}$ E. Unger,${ }^{1}$ and E. Turkof. ${ }^{2}{ }^{1}$ Department of Biomedical Engineering and Physics and ${ }^{2}$ Department of Plastic and Reconstructive Surgery, University of Vienna, Vienna, Austria 
Intraoperative electroneurodiagnostic is a diagnostic tool used during surgery of peripheral nerves to precisely localize the site of lesions or to determine the functional status of the exposed nerve segments. In principle, the nervous structure to be investigated is stimulated at its most proximal (healthy) site, thereby evoking an efferent nerve compound action potential that is recorded from the surface of the nerve at the exposed site. Stimulation sites vary according to the structure to be investigated. For brachial plexus lesions, we perform motor evoked potentials (transcranial electrical stimulation of the motor cortex) as we do for scoliosis surgery. For peripheral nerve lesions, we stimulate the spinal roots of the damaged nerve with surface electrodes placed paravertebrally above the respective nerve segments, and for unknown sites of facial nerve lesions we stimulate the facial nerve transcrianally at its exit from the pons. Subsequent to the electrical stimulus, the evoked nerve action potential's shape we conclude to be the function of the nerve. The amplitude of the electroneurogram (ENG) is proportional to the number of functioning nerve fibers. Former measurements have been done using commonly used 2 channel electromyography with self-constructed, special prototype electrodes in combination with a commercially available stimulator (Digitimer 185). The Digitimer can deliver monophasic rectangular impulses with a fixed pulse width of $50 \mu$ s amplitudes of up to $1,000 \mathrm{~V}$. The findings using this equipment, like mains dependency, fixed pulse width, only 2 channel recording, and the impossibility of short latency ENG recordings due to overdriving the recording amplifier led to the development of a 6 channel mobile (laptop PC), flexible, and safe (battery powered) system with newly designed multichannel recording electrodes for reliably reproducible measurements combined with an integrated adjustable biphasic electrical stimulator. The preamplifier is equipped with a crowbar input protection circuit caused by the high stimulation amplitude (up to $1,000 \mathrm{~V}$ ) and it recovers after overdriving within $1 \mathrm{~ms}$. To amplify signals in the range of $10 \mu \mathrm{V}$ with a bandwidth of $10 \mathrm{kHz}$ a high signal to noise ratio is required. The stimulator is variable in amplitude up to $1,000 \mathrm{~V}$ and pulse width from $50 \mu \mathrm{s}$ to $1 \mathrm{~ms}$. Furthermore the discharge phase of the impulse is separately adjustable in duration to minimize stimulus artifact. The recording is triggered by the stimulation pulse and is active for 100 $\mathrm{ms}$; thus, it can be (together with parameters) comparable stored in a database. Without important loss of time the system is rapidly applicable in the operating room because of its safe and easy handling.

STIMULATION OF NERVES INNERVATING THE DOG PANCREAS. J. Rozman, ${ }^{1}$ B. Zorko ${ }^{2}$ M. Bunc,${ }^{3}$ and ${ }^{4}$ M. Žitko. ${ }^{1}$ ITIS d. o. o. Ljubljana, Center for Implantable Technology and Sensors, University of Ljubljana; ${ }^{2}$ Veterinary Faculty; ${ }^{3}$ School of Medicine, Institute of Pathophysiology; and ${ }^{4}$ Department of Nuclear Medicine, University Medical Center Ljubljana, Ljubljana, Slovenia

The purpose of the study was to modulate secretion of insulin and glucagon into the blood of a healthy and diabetic dog with stimulation of nerves innervating the pancreas. Six 33 electrode spiral cuffs were fabricated and implanted in an adult beagle dog (weight $15 \mathrm{~kg}$ ). The first cuff was installed on the vagus nerve at the neck, the second on the splanchnic nerve before the celiac ganglion, and the last cuff on the pancreatic nerve at the site just before it enters the pancreas. Partial destruction of islet $\beta$-cells in the pancreas, to cause insulin-dependent Type 1 diabetes, was induced with Alloxan. A quantity of Alloxan, calculated according to the weight of the animal, was intravenously injected into the blood. After $24 \mathrm{~h}$ the pancreatic $\beta$-cells were irreversibly damaged and permanent hyperglycemia was induced. Then insulin therapy was applied. In experiments each nerve was stimulated using biphasic, rectangular current pulses with parameters of width 200 $\mu \mathrm{s}$, amplitude adjustable between 10 and $20 \mathrm{~mA}$, and frequency 20 Hz. Electrocardiogram, blood pressure, and pressure in the bladder were recorded to evaluate the influence of stimulation on the function of the cardiovascular system, lungs, and urinary tract.
Samples from the femoral artery were drawn each time before any experimental activity to measure the level of glucagon, insulin, and C-peptide in the blood. In all stimulations, blood samples from the femoral artery were drawn at the beginning, after 5 min of stimulation, and $5 \mathrm{~min}$ after the end. They were placed on ice, centrifuged at $4{ }^{\circ} \mathrm{C}$ immediately after the end of the experiments, and the plasma was separated and frozen at $-20^{\circ} \mathrm{C}$ until radioimmunological assay (RIA). In a series of stimulations using the described stimuli, where steps of current pulses were between 10 and $20 \mathrm{~mA}$, measurable changes were observed in splanchnic and in vagus nerves, respectively. Splanchnic nerve stimulation increased bladder pressure and precipitated generalized stomach contractions. Drastic changes were observed during stimulation of the nerve vagus, expressed as a rapid decrease of arterial blood pressure and irregular heart rate. Further increase of the vagal stimuli caused brachicardia then asystolia and hypotension. Results of RIA of blood samples confirmed the hypothesis that in intact pancreas stimulation of the vagus nerve caused a considerable increase in insulin secretion. However, vagal nerve stimulation did not cause significant changes in glucagon secretion. Vagal nerve stimulation caused also some decrease in C-peptide secretion. Results also showed that in intact pancreas splanchnic nerve stimulation did not cause considerable change in insulin secretion while considerable increse in glucagon secretion was noticed. Splanchnic nerve stimulation did not cause considerable change in C-peptide secretion. Pancreatic nerve stimulation in intact pancreas did not change considerably the secretion in any of the 3 hormones. Results showed that in severed pancreas vagal nerve stimulation caused an increase in insulin secretion. Moreover, vagal nerve stimulation also increased glucagon secretion. Besides, vagal nerve stimulation caused a minor increase in C-peptide secretion. In severed pancreas, splanchnic nerve stimulation caused a minor decrease in insulin secretion and a considerable increase in glucagon secretion. In severed pancreas, splanchnic nerve stimulation caused a small increase in C-peptide secretion. In severed pancreas pancreatic nerve stimulation did not cause considerable change in insulin secretion while minor increase in glucagon and C-peptide secretion was observed.

OBJECT ORIENTED SOFTWARE COMPONENTS FOR MULTICHANNEL-STIMULATOR CONTROL. M. Russold, M. Bijak, M. Reichel, S. Sauermann, and W. Mayr. Department of Biomedical Engineering and Physics, University of Vienna, Vienna, Austria

In the past a PC-based software package was used to set up the stimulation parameters for an 8 channel surface stimulator, mainly used for paraplegic subjects' reactivation of lower extremities. To simplify the design of future applications, a set of flexible object oriented software components was created. These components allow us to manipulate the stimulation parameters in various ways. Additionally, these tools can be adapted and extended easily according to the project specific needs. Use in different development environments (e.g., MS Visual C++, MS Visual Basic, and so on) is possible in a straightforward way. After a detailed functional analysis 4 components were realized: a graph component, a pulse component, a security component, and a database component. Each of them contains a set of functions, procedures, and properties to fulfill their required tasks. The graph component allows a mouse-controlled manipulation of the burst envelope in an easy and intuitive way, continuing in the pulse component, which generates stimulator control sequences. A simple hierarchical user management is supported by the security component. For storage of stimulation parameters and measurement results, as well as for later data analysis, a relational database structure is implemented. Communication with this database and all data handling is established by means of the database component. The components are combined in a sample application to demonstrate their proper use. After an extensive testing phase, a first patient approach began at the Department of Physical Medicine and Rehabilitation. Concluding modifications are 
considered and intended to be finished by the time of this workshop.

AUTOMATED ESTIMATION OF STIMULATION THRESHOLDS USING SINGLE TWITCHES AND ACCELERATION SENSORS. S. Sauermann, M. Bijak, M. Reichel, D. Rafolt, E. Unger, W. Mayr, and H. Lanmüller. Department of Biomedical Engineering and Physics, University of Vienna, Vienna, Austria

One of the first tasks in achieving movements by functional electrical stimulation (FES) is the estimation of the relation between stimulation input and muscle force output. This relation is often called the recruitment curve. Especially with surface stimulation systems the properties and position of stimulation electrodes have strong influence on the recruitment curve. In the clinical application it therefore has to be established at every session and even within sessions. This estimation process is of clinical relevance as it takes time and effort that would be better invested in optimization of the stimulation outcome. This work was therefore aimed at a method for automated estimation of the stimulation threshold in the clinical application of FES systems. It uses low frequency ramp stimulation in order to generate a series of muscle twitches with increasing amplitude. An acceleration sensor is used to measure these twitches. A signal analysis algorithm then evaluates the resulting spikes within the acceleration signal. Spikes below a given signal-to-noise level are discarded as well as spikes out of a given time window around the stimulation period. Together with the known number of impulses and the stimulation amplitude at the start and the end of the stimulation ramp an amplitude interval is then calculated that contains the stimulation threshold. The number of spikes found by the algorithm was compared to that found by an experienced observer. The control software for stimulation and measurement and the analysis algorithm were all implemented on a laptop PC. A test on the quadriceps femoris muscle of 21 healthy test persons was performed. The acceleration sensor was attached to the skin above the bulk of the muscle with double stick tape. Bursts of 10 biphasic impulses of $150+150 \mu$ s impulse duration at $10 \mathrm{~Hz}$ were applied via surface electrodes. The automated measurement equipment proved very useful in speeding up the measurement process. The algorithm estimated the number of spikes correctly in 15 of 21 trials. It missed a single spike in 2 cases and detected 1 wrongly in 1 case. In the remaining 3 cases the number of spikes was wrong by more than 1. Further work now will be directed at more sophisticated signal analysis based on the dataset of signals harvested in this trial and improved sensing methods. The acceleration sensors provided signals of sufficient quality at a reasonable effort for setting them up. Nevertheless the necessary amount of cabling has to be reduced significantly. It also will be of primary concern to establish the accuracy of the recruitment curve that is necessary to achieve satisfying stimulation outcome with the intended applications.

FIRST INSIGHTS ON MUSCLE AFFERENT ELECTRONEUROGRAM AND NEURAL NETWORKS FOR CLOSED LOOP CONTROL OF FUNCTIONAL ELECTRICAL STIMULATION GENERATED RABBIT ANKLE MOVEMENTS. F. Sepulveda, W. Jensen, and T. Sinkjær. Center for Sensory-Motor Interaction, Aalborg University, Aalborg, Denmark

Control of functional electrical stimulation based movements can be enhanced by proper estimation of the current state of motion. Some of the latter information can be obtained from muscle afferent fibers. As such, a simple approach has been taken so far by our group: rectified and bin-integrated electroneurogram (ENG) signals have been monitored and have been found to allow for a reasonable mapping onto angular and torque data by means of neural and fuzzy models. This article presents our first findings from using the extracted angular information in a closed-loop controller. Acute experiments were conducted with rabbits. For extraction of the ENG signals, tripolar cuff electrodes were implanted onto the peroneal and tibial branches of the sciatic nerve in the left hind limb. To minimize cutaneous inputs, the sural nerve was transected distal to its origin in the tibial nerve. The tibial and peroneal nerves were also transected just above the ankle joint to minimize sensory inputs from the foot. The ENG signals were sampled at $10 \mathrm{kHz}$ for $4 \mathrm{~ms}$ before each stimulation pulse (to eliminate stimulation artifacts). A neural network (trained off-line) was used for extraction of joint angles from the ENGs. Inputs for the neural network were the mean ENG values over the above $4 \mathrm{~ms}$ period. For stimulation purposes, percutaneous stainless steel wires were placed intramuscularly into the tibialis anterior and lateral gastrocnemius muscles, respectively. Stimulation intensity was varied by changing the applied pulse width. Step and sinusoidal tracking tasks were performed using a standard proportional-integral derivative (PID) controller. Early results showed that the system's performance is highly sensitive to the initial joint angle. Best results were obtained when starting with the ankle joint at a neutral rest angle. Furthermore, angles estimated from the ENG and neural networks lost correlation with measured angles as a given experiment progressed. Improvements were seen when the neural networks were allowed to learn intermittently during an experimental session. Finally, a standard PID controller required frequent retuning during an experimental session, which, not surprisingly, suggests that an adaptive controller should be used instead.

OUR EXPERIENCE WITH SLOW PULSE STIMULATION IN PATIENTS WITH POSTTRAUMATIC NEUROPATHIES. J.K. Terzis and W.T. Liberson. Eastern Virginia Medical School, Norfolk, VA, U.S.A.

We share our experience with slow purse stimulation of denervated skeletal muscle in patients that underwent microsurgical repair following devastating lesions of the brachial plexus in obstetrical paralysis and facial paralysis. A slow pulse stimulator was developed by 1 of the authors (W.T. Liberson) that permits a rational technique for treating denervated muscle. First, slow pulses are calibrated as to their duration so that the latter may be chosen for each individual patient according to his needs. The pulses are delivered automatically at a rate that may be harmless. We are now satisfied with the fact that stimuli succeeding each other at a rate of 1 during each $10 \mathrm{~s}$ do not harm the skin if applied for a period of 20 to $30 \mathrm{~s}$. The stimulator has a timer limiting sessions to $20 \mathrm{~min}$. In areas where denervated fibers may be mixed with normal muscle fibers, to avoid stimulation of the normal muscles the use of progressive onset of the stimulating pulses has been successfully employed. A time of onset on the order of 100 or $200 \mathrm{~ms}$ indeed suppresses the contraction of the innervated muscles. We limit the total time of stimulation to $5 \mathrm{~h}$ for adults and $3 \mathrm{~h}$ for children. The treatment sessions are $20 \mathrm{~min}$ each with an interval of $1 \mathrm{~h}$ in between. The patient is warned never to restimulate an area that remains red following the previous session of stimulation. We have used this type of slow pulse stimulation for the past 15 years in all our patients that have undergone microsurgical reconstruction of the injured brachial plexus, in cases of obstetrical brachial plexus palsy, and in selected cases of facial paralysis. Outcomes of microreconstruction all combined with this form of slow pulse electrotherapy will be presented and benefits outlined. Our technique allows simultaneous stimulation of practically all upper extremity muscles by placing 1 electrode on the shoulder and the other on the palmar aspect of the fingers. Although the wisdom of stimulation of denervated muscles often has been challenged, we believe strongly that this does not apply to human denervated muscle. Until new therapeutic procedures are developed to compensate for the loss of the nutrient axon slow 
pulse electrotherapy is an effective alternative for the treatment of denervated muscle.

AUTOMYOELECTRIC CONTROL OF FUNCTIONAL ELECTRICAL STIMULATION ON THE TIBIALIS ANTERIOR: A PILOT STUDY WITH STROKE PATIENTS. Rune Thorsen, ${ }^{1,2,3,4}$ Jane Burridge,${ }^{5}$ Nick Donaldson, ${ }^{3}$ Maurizio Ferrarin, ${ }^{1}$ Jonathan Norton, ${ }^{3}$ and Peter Veltink. ${ }^{5}{ }^{1}$ Centro di Bioingegneria, Fond. Don Gnocchi-Politecnico di Milano, Milan, Italy; ${ }^{2}$ Salisbury District Hospital, Salisbury; ${ }^{3}$ University College London, London, U.K.; ${ }^{4}$ University of Twente, Enschede, The Netherlands; and ${ }^{5}$ University of Southampton, Southampton, U.K.

In this study of 9 subjects with dropped foot as a result of hemiplegia following stroke electromyographic (EMG) signals from the tibialis anterior (TA) muscle were used to control functional electrical stimulation (FES) of the same TA muscle group. The technique has been termed automyoelectric control of FES (AutoMeCFES). EMG signals were recorded using surface electrodes and processed digitally to allow continuous control of the amplitude of FES applied to the common peroneal nerve. Isometric force of dorsiflexion was measured with the patient sitting and the leg hanging freely with the knee at approximately $90^{\circ}$. A tracking test was used to compare the control and amplitude of the ankle torque with and without stimulation. Seven of the nine patients had increased torque amplitude of whom $3 \mathrm{had}$ increased control with the system. An immediate carryover effect was seen in 1 subject. It is concluded that for selected patients AutoMeCFES can increase the muscle force of TA without compromising control.

GENERATION OF NEW WAVEFORMS TO ACHIEVE ANODAL BLOCK: A COMPUTER STUDY. Arantxa Uranga ${ }^{1}$ and Nico J.M. Rijkhoff. ${ }^{2}{ }^{1}$ Dept. Enginyeria Electrònica, Universitat Autònoma de Barcelona, Barcelona, Spain; and ${ }^{2}$ Center for Sensory-Motor Interaction, Aalborg University, Aalborg, Denmark

Electrical nerve stimulation is used to restore control of different organs. However, electrical stimulation results in an inverse recruitment order of nerve fibers since large fibers are activated before smaller ones. As a consequence, fast onset of muscle fatigue and poor force gradation are some drawbacks. Moreover, some applications, such as electromicturition and electrodefecation, require activation of small fibers without activation of the larger ones. Different methods have been proposed to achieve a more natural recruitment order such as selective anodal block, slowly rising pulses, and high frequency block. We focused in this study on the anodal block technique. This technique takes advantage of the different excitation and blocking thresholds for small and large fibers. It is possible with this technique, using a combination of excitation and selective blocking, to activate primarily the small diameter nerve. In order to obtain anodal blocking, relative large pulse widths and amplitudes are used compared with the traditional stimulation pattern. This induces a larger charge injection, which can be harmful not only to the tissue but also to the electrode. The present study tries to develop new pulse shapes so that injected charge could be reduced. Because the action potential needs time to travel from the cathode to the anode, strong hyperpolarization is not needed at the beginning of the pulse. The use of pulse shapes with 2 different amplitude levels (a smaller amplitude at the beginning of the pulse) and with an increasing slope has been simulated in a computer model and the reduction in the amount of charge injected has been evaluated. In addition, the effect of the use of a hyperpolarizing prepulse on the excitation and blocking thresholds has been analyzed. Parameters such as hyperpolarizing pulse amplitude, pulse width, and delay have been optimized to obtain a charge reduction. Based on this study we conclude that anodal blocking can be obtained with new waveforms that allow for a reduced charge injection. The use of these shapes will be safer in chronic applications of anodal blocking.

TOWARD AUTOMATIC OPTIMIZATION OF GAIT SUP. PORT BY A TWO CHANNEL IMPLANTABLE DROP FOOT STIMULATOR FOR STROKE PATIENTS. Peter $H$. Veltink, ${ }^{1}$ Johan Hemssems, ${ }^{1}$ Per Slycke,${ }^{2}$ Edwin Morsink,${ }^{2}$ Gerrit Bultstra, ${ }^{1}$ and Hermie Hermens. ${ }^{3}{ }^{1}$ Institute for Biomedical Technology, University of Twente; ${ }^{2}$ Xsens Sports Technologies bv; and ${ }^{3}$ Roessingh Research and Development bv, Enschede, the Netherlands

Stroke may have many physical and cognitive consequences. Physically, the motor control of 1 side of the body may be deteriorated. Often, such a deteriorated motor function improves during the recovery period and by training, although in many cases motor control problems remain. Among others, a frequent consequence is the inability to voluntarily lift the foot at the affected side (drop foot). Since Liberson's work (1961), functional electrical stimulation systems have been developed to activate artificially the dorsiflexor muscles during the swing phase of gait. Recently, a 2 channel implantable drop foot stimulator was developed (Holsheimer et al., 1993) and applied in patients, allowing electronic balancing of inversion and eversion during foot lift. In the current project, an automated system was developed to optimize the timing and magnitude of stimulation of both stimulation channels on the basis of criteria for the quality of movement of the foot during the swing phase of gait. These criteria encompass the timing of the start of dorsiflexion movement after heeloff, maximal dorsiflexion, balance between inversion and eversion and height of foot lift during midswing, and controlled foot placement (heel first) at the end of the swing phase. These criteria are evaluated using a 6 degrees of freedom inertial sensor system placed on the foot. On the basis of this evaluation the timing and magnitude of the stimulation are adapted from cycle to cycle. The observability of the formulated movement criteria from the inertial sensor measurements have been evaluated preliminarily in a patient experiment and an analysis of the controllability of the foot movement judged by the criteria is being performed. The approach is intended to result in an evaluation and tuning system for clinical use.

PEAK PHYSICAL WORK CAPACITY DURING ARM ERGOMETRY, FUNCTIONAL ELECTRICAL STIMULATION CYCLING, AND TWO HYBRID EXERCISE CONDITIONS IN SPINAL CORD INJURED PATIENTS. Joeri Verellen, ${ }^{1,2}$ Bradford Kerby, ${ }^{1}$ Bohdan Olijnyk, ${ }^{1}$ Souheil Saab, ${ }^{1}$ Gordon Smith, ${ }^{1}$ Robert D. Steadward, ${ }^{1}$ Yves Vanlandewijck, ${ }^{2}$ Brian Andrews, ${ }^{3}$ and Garry D. Wheeler. ${ }^{1}$ The Steadward Centre, Faculty of Physical Education and Recreation, University of Alberta, Edmonton, Alberta, Canada; ${ }^{2}$ Faculty of Physical Education and Physiotherapy, Catholic University of Leuven, Leuven, Belgium; and ${ }^{3}$ University of Oxford, Oxford, and The Stoke Mandeville Hospital, National Spinal Injuries Unit, Aylesbury, U.K.

Interest in enhancing exercise opportunities, and electrostimulation assisted exercise in particular, for the spinal cord injured (SCI) has been mediated by evidence of increased risk for heart disease in this population. The purpose of this study was to compare a newly developed functional electrical stimulation (FES) assisted rowing machine (ROWSTIM) with arm ergometry (ACE), FES cycling (CFES LE), and hybrid cranking and cycling (CFES LE + ACE). Five SCI participants (C7-T12) underwent a progressive maximal peak oxygen assessment to ascertain peak physical work capacity across 4 conditions with 3 trials per exercise modality to establish reliability of measurement. Peak functional aerobic capacity and heart rates were correlated significantly across repeated tests for ACE, CFES LE, and ACE + CFES LE. Tests 1 and 2 of FES rowing were significantly corre- 
lated, however Tests 1 and 3 , and 2 and 3 , were not $(\mathrm{p}<0.01)$. A one-way ANOVA with condition as the main factor showed peak absolute and relative functional aerobic capacity and heart rate to be significantly lower for CFES LE versus absolute and relative ACE, CFES LE + ACE, and FES ROW measures $(\mathrm{p}<0.05)$. However, there were no significant differences between both hybrid exercise conditions or between hybrid exercise and arm ergometry. Preliminary results suggest that the ROWSTIM is as effective as an exercise device or training tool for the SCI as FES cycling or hybrid cycling and cranking. However, a larger sample size and further technological developments of the ROWSTIM are needed to demonstrate the efficacy of rowing over upper extremity exercise and hybrid cycling and cranking.

FUNCTION OF SKELETAL MUSCLE TISSUE FORMED AFTER MYOBLAST TRANSPLANTATION INTO IRRADIATED MOUSE MUSCLES. A. Wernig, M. Zweyer, and A. Irintchev. Department of Physiology, University of Bonn, Bonn, Germany

Pretreatment of host muscles with ionizing radiation enhances the formation of donor-derived tissue after myoblast transplantation in the mouse, but there is little evidence for improvement of muscle function. To investigate this, we implanted myoblasts from an expanded, male-donor derived, primary culture (i28) into x-ray irradiated $(16 \mathrm{~Gy})$ or irradiated and damaged soleus muscles of female syngenic mice $(\mathrm{Balb} / \mathrm{c})$. Three to six months later the isometric contractile properties of transplanted and control muscles were studied in vitro, and the Y chromosome positive progeny of the implanted cells was visualized on muscle cross sections. Irradiated and vehicle-injected muscles had significantly smaller weights than untreated solei and produced less twitch and tetanic tension (all about 18\%). Such deficits were not found in irradiated solei implanted with $10^{6}$ myoblasts. Increase of muscle mass and strength was due to the integration of donor-derived cells. No deficits in nerve-evoked tension were found. Repeated freezing/ thawing in situ of irradiated muscles led to formation of soleus remnants devoid of or containing only small amounts of contractile tissue (1-50 muscle fibers). Myoblasts $\left(10^{6}\right)$ implanted into such destructed muscles generated numerous muscle fibres (1,200-5,000 per muscle). Upon direct electrical stimulation these fibers produced considerable twitch (53\% of normal) and tetanic tensions $(35 \%)$. The newly formed muscles were, however, insufficiently innervated presumably due to radiation mediated arrest of Schwann cells. Separate studies on nerve regeneration following $\mathrm{x}$-irradiation and nerve crush or botulinum application will be demonstrated. In any case, even after complete muscle destruction the disorganized suspension of donor cells will produce new organized contractile tissue thus replacing the host muscle. A limiting factor is the reduced capacity of the nerve to regenerate after $\mathrm{x}$-irradiation. Apart from these results, the role of the satellite cell in muscle fiber repair as well as being a possible source for pluripotent stem cells is discussed. The deviating behavior of human myogenic cells in their limited proliferative capacity and telomere length related senescence is mentioned.

PRINCIPLES OF LAUFBAND THERAPY FOR SPINAL CORD DAMAGED PERSONS. A. Wernig and S. Müller. Department of Physiology, University of Bonn, Bonn; and Klinikum Karlsbad-Langensteinbach, Karlsbad-Langensteinbach, Germany

Locomotor training on the Laufband focuses on intensive walking in an upright position, facilitated by body weight support via a harness, the moving band of the treadmill, and initial limb setting by 2 therapists if necessary. These principles are derived from observations in spinal animals on activity-related learning of the isolated spinal cord and on the rules of spinal locomotion in lower vertebrates. The novel approach to train intensely precisely that skill that needs to be achieved (upright walking) allows us to reach therapeutic goals far beyond those possible by conventional phys- iotherapy. As a rule in motor rehabilitation, therefore, each patient with some if little voluntary muscle activity remaining needs to be trained on the Laufband to find and approach his individual limits of locomotion. Practical obstacles for a successful application of Laufband therapy on a broader scale at the time are the poor education of the therapists in practical handling of the patients and in their imagination of the possible therapeutic goals to be reached.

WHEN CAN AUTOCORRELATION BE USED FOR THE CLASSIFICATION OF ELECTRONEUROGRAM SIGNALS? L. Zhou, and N.de.N. Donaldson. Implanted Devices Group, University College London, London, U.K.

It has been shown that it is possible to pick up nerve signals using chronically implanted tripolar electrode cuffs and that these signals are stable. However, some nerves, which carry signals of use for neuroprostheses, also carry other signals that we would like to distinguish. We wish to separate the bladder signals from those originating from the cutaneous afferents and from the rectum. Jezernik suggested that this could be done by autocorrelation function (ACF), exploiting the fact that their tripole action potentials are different because they are carried on fibers of different diameters. The signals, seen at the sacral root, are very small compared to the noise. So the first question is at what level can we distinguish the bladder signal when it is buried in noise? The effect of noise was investigated by simulation. Artificially generated electroneurogram (ENG) signals from each origin and noise were mixed and passed through filters with various passbands. From the ACF of the pure bladder signal a range of delay was chosen within which the first negative peak occurred, and this was used as the detection range for bladder signals. A decision must be made about the passband for the amplifier. Making the band narrow reduces the noise power (the noise is white at source). However, filtering the white noise produces a random signal that becomes similar to the signal as the band gets narrower and that has a similar ACF. One would therefore expect an optimal passband at which signals with the lowest signal-to-noise ratio (SNR) are distinguishable. We applied the limiting criterion that at least $50 \%$ noisy bladder ENGs are correctly detected. For 3 filters, the results are (1) filter passband 100 to $10,000 \mathrm{~Hz} /-6 \mathrm{~dB}$ limiting SNR, (2) 100 to $5,000 \mathrm{~Hz} /-8 \mathrm{~dB}$, and (3) 100 to $2,500 \mathrm{~Hz} / 0$ $\mathrm{dB}$. With the best passband $(100-5,000 \mathrm{~Hz})$ the error rate for detecting bladder signal falls below $50 \%$ when the SNR is only -8 $\mathrm{dB}$. As the power density spectrum of the synthesized bladder ENG signal has fallen to half its peak power at $2,000 \mathrm{~Hz}$, this also shows that the amplifier passband should be significantly wider than the signal spectrum. Whether actual signals recorded from cuffs on the sacral nerve roots have an SNR as high as $\sim-8 \mathrm{~dB}$ seems doubtful, based on existing knowledge. Therefore, autocorrelation may not be a usable method in this application.

\section{Upcoming Meetings}

\section{8th Annual Meeting and Exposition of the Society for Biomaterials}

April 24-27, 2002, Tampa, Florida. For more information, contact Society for Biomaterials, 13355 Tenth Avenue North, Suite 108, Minneapolis, MN 55441-5510. Tel: 763543-0908; FAX: 763-765-2329; Web: http://www.@ biomaterials.org. E-mail: registration@biomaterials.org

\section{ASAIO'S 48th Annual Conference}

June 13-15, 2002, New York. For more information, contact ASAIO Headquarters, P.O. Box C, Boca Raton, Florida 33429-0468. Tel: 561-391-8589; FAX: 561-3689153. E-mail: info@asaio.com 


\section{9th International Congress of the}

\section{Transplantation Society}

August 18-23, 2002, Buenos Aires, Argentina. For more information, contact CONGRESS SECRETARIAT, ana juan congresos, Sarmiento $15624^{\circ} \mathrm{F}$ (C1042ABO) Buenos Aires, Argentina. Tel: (54-11) 4381-1777; FAX: (54-11) 4382-6703. E-mail: info@transplantation2002.com Web: www.transplantation2002.com

\section{European Society for Artificial Organs}

August 28-31, 2002, Vienna, Austria. For more information, contact Scientific Secretariat, Institute of Biomedical Research, c/o Mrs. Eva Klausner, Waehringer Guertel 1820, A-1090 Vienna-Austria. Tel.: +43-1-40400 5221. Fax: +43-1-40400 5229. E-mail: esao2002@akh-wien.ac.at Website: http://www.akh-wien.ac.at Congress President: Udo M. Losert 9th Congress of the World Apheresis Association

September 6-10, 2002, Paris, France. For more information, contact, WAA 2002 c/o Colloquium, 12, rue de la Croix Faubin, F-75557 Paris cedex 11, France. Tel: 33-14464-1515; FAX: 33-1-4464-1516; E-mail: waa2002@ colloquium.fr; Site web: www.colloquium.fr

\section{0th Congress of the International Society for Rotary} Blood Pumps

September 11-14, 2002, Osaka, Japan. For more information, contact Congress Office, 10th Congress of the International Society for Rotary Blood Pumps, Department of Surgery (El), Osaka University Graduate School of Medicine, 2-2 Yamada-oka, Suita, Osaka 565-0871, Japan. Tel: +81-6-6879-3151; FAX: +81-6-6879-3159; E-mail: isrp@surg1.med.osaka-u.ac.jp; Homepage: http://www. osaka-u.ac.jp/pub/surg1/isrp 